\title{
Bioactive and Nutritional Potential of Medicinal and Aromatic Plant (MAP) Seasoning Mixtures
}

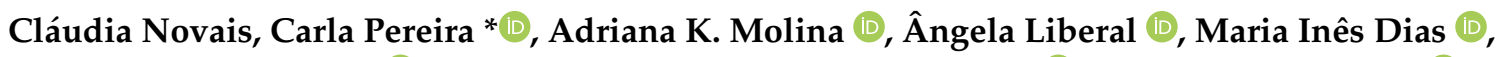 \\ Mikel Añibarro-Ortega (D, Maria José Alves, Ricardo C. Calhelha (D), Isabel C.F.R. Ferreira (D) and Lillian Barros *(D) \\ Centro de Investigação de Montanha (CIMO), Instituto Politécnico de Bragança, Campus de Santa Apolónia, \\ 5300-253 Bragança, Portugal; a32125@alunos.ipb.pt (C.N.); amolina@ipb.pt (A.K.M.); angela.liberal@ipb.pt (Â.L.); \\ maria.ines@ipb.pt (M.I.D.); mikel@ipb.pt (M.A.-O.); maria.alves@ipb.pt (M.J.A.); calhelha@ipb.pt (R.C.C.); \\ iferreira@ipb.pt (I.C.F.R.F.) \\ * Correspondence: carlap@ipb.pt (C.P.); lillian@ipb.pt (L.B.); Tel.: +351-2733-309-04 (C.P.); +351-2733-309-01 (L.B.)
}

check for updates

Citation: Novais, C.; Pereira, C.; Molina, A.K.; Liberal, Â.; Dias, M.I.; Añibarro-Ortega, M.; Alves, M.J.; Calhelha, R.C.; Ferreira, I.C.F.R.; Barros, L. Bioactive and Nutritional Potential of Medicinal and Aromatic Plant (MAP) Seasoning Mixtures. Molecules 2021, 26, 1587. https:// doi.org/10.3390/molecules26061587

Academic Editor: Francesco Cacciola

Received: 24 February 2021

Accepted: 11 March 2021

Published: 13 March 2021

Publisher's Note: MDPI stays neutral with regard to jurisdictional claims in published maps and institutional affiliations.

Copyright: (c) 2021 by the authors. Licensee MDPI, Basel, Switzerland. This article is an open access article distributed under the terms and conditions of the Creative Commons Attribution (CC BY) license (https:// creativecommons.org/licenses/by/ $4.0 /)$.

\begin{abstract}
Medicinal and aromatic plants (MAPs), worldwide appreciated and used as condiments, dyes, and preservatives, possess several biological properties that justify their continuous application in the food industry. In the present study, the nutritional and chemical profiles, as well as the bioactive properties of four combinations of condiments, sold for seasoning poultry, meat, fish, and salads, were evaluated. Twenty-five phenolic compounds (HPLC-DAD-ESI/MS) were identified, with apigenin-O-malonyl-pentoside-hexoside as the major compound detected in all extracts. Oxalic and citric acids were identified in all mixtures (UFLC-PDA), as well as all the four tocopherol isoforms (HPLC-fluorescence). Regarding bioactivities, the mixtures for meat and salads (TBARS) and meat and poultry (OxHLIA) stood out for their antioxidant potential, whereas for the anti-inflammatory and antitumor properties, the mixtures revealing the greatest results were those for poultry and salad, respectively. In terms of antimicrobial activity, all the mixtures revealed the capacity to inhibit the growth of some bacterial strains. In brief, condiment mixtures showed to be a good source of bioactive compounds, as they confer health benefits, validating the importance of their inclusion in the human diet as a good dietary practice.
\end{abstract}

Keywords: condiment mixtures; phenolic compounds; organic acids; tocopherols; bioactivities; nutritional value

\section{Introduction}

The emergence of new diseases, degenerative conditions, and health problems related to physical inactivity, coupled with an increased life expectancy, have led to a growing intake of medicines. Nevertheless, the occurrence of several adverse effects related to the indiscriminate use of synthetic drugs has boosted the search for new and less aggressive treatments, often complementary to conventional ones [1]. Currently, herbal medicines are considered as low-cost preventive agents for various health issues, presenting low or practically non-existent toxicity, a high and proven effectiveness, the possibility of being taken orally, a well-known mechanism of action, and a good acceptance by the general and scientific community [2]. On the other hand, these plants capacity to prevent foodborne diseases and promote the extension of food shelf-life has led to their exploitation as sources of bioactive compounds to be employed in food industry, which mostly relies on artificial additives.

Since ancient times, spices and MAPs have played an important role in human's nutrition, imparting aroma, flavor, and color to food. Through their use as condiments, it is possible to reduce the use of salt and fat and, therefore, they are gaining increasing importance in good dietary practices [3]. Known as functional foods, these herbs not only meet common nutritional requirements, but also provide physiological benefits, being 
mostly composed of carbohydrates and a wide range of sugars, usually in low amounts, but also possessing a unique richness in other bioactive molecules $[4,5]$.

Different groups of compounds are known to be present in MAPs, many of them having pharmacological properties, such as tocopherols and phenolic compounds, among others [4]. These latest have been considered one of the most important groups of natural and chemopreventive antioxidants, with potential anti-inflammatory, antidiabetic, and anticancer effects, and with cardio, hepatic, and neuroprotective actions, thus playing a crucial role in human health [6-9]. More importantly, these properties can be influenced by the action of other plant constituents, which can result in enhanced biological activities through synergistic or additive effects [10]. Together, these properties are of great interest in food, pharmaceutical, cosmetic, and other industries, promoting the health of the general population, both through their direct use in food and through their incorporation into different products, making them functional and biodynamic [11-13]. In this field, recent studies emphasize the efficiency of MAPs extracts in the development of bakery and dairy products, with functional properties for consumers' health improvement [13-15]. At a more specific level, these plants can also be used as complementary therapies in the treatment of diabetes mellitus, given their recognized antidiabetic activity through different mechanisms of action [16], in inhibiting the proliferation of tumor cells, targeting enzymes and DNA transcription factors $[17,18]$, and being important allies in the maintenance of human immune system, due to the presence of compounds with recognized anti-inflammatory potential [19].

Given these features, there is a global trend toward the development of herbal medicines from different mixtures of natural products, aiming at the standardization and a synergistic evaluation of the bioactive compounds present in these products [20-22]. On the other hand, given their widely appreciated taste, several mixtures have been prepared for seasoning purposes by a Portuguese company, using specific herbs that are more appreciated when applied to meat, poultry, salads, or fish. The plants selected for these mixtures' preparation were Allium schoenoprasum L., Petroselinum crispum L., Salvia officinalis L., Satureja montana L., Thymus vulgaris L., Thymus mastichina L., Rosmarinus officinalis L., Origanum vulgare L., Artemisia dracunculus L., and Thymus $\times$ citriodorus L. Each mixture contains different proportion of 4 to 5 plants, according to their specific taste and traditional use. Thus, and given their wide use in Portuguese cuisine, the aim was to characterize these four mixtures of condiments both chemically and nutritionally and evaluate their bioactive properties, corroborating the importance of their inclusion in daily diet and in complementary therapeutic applications.

\section{Results and Discussion}

MAPs are worldwide established as nutritious plants commonly present in the Mediterranean diet. In the present study, four mixtures of condiments were assessed for their nutritional, chemical, and bioactive properties. These mixtures were created and are commercialized by the Portuguese company Cantinho das Aromáticas. The plants were selected and combined according to their specific taste and traditional use, but their relative proportion in the mixtures is protected for commercial reasons. In terms of qualitative composition, the mixture for poultry consists of Allium schoenoprasum L., Petroselinum crispum L., Salvia officinalis L., Satureja montana L., and Thymus vulgaris L.; the mixture for meat is composed of P.crispum, S. officinalis, Thymus mastichina L., and Rosmarinus officinalis L.; the mixture for salads consists of A. schoenoprasum, P. crispum, Origanum vulgare L., and T. mastichina; and the mixture for fish is composed of A. schoenoprasum, Artemisia dracunculus L., P. crispum, and Thymus $\times$ citriodorus $\mathrm{L}$.

\subsection{Nutritional Value}

The results reached for macronutrients of the studied mixtures are shown in Table 1. Since all the analyzed condiments are mixtures of plants whose only qualitative composition is known and no information is provided regarding their proportion in each mixture, 
the comparison of the results obtained was made, whenever possible, considering the available literature for the individual plants. Carbohydrates were the major macronutrients found in all mixtures, with values ranging from 65.2 to $72.0 \mathrm{~g} / 100 \mathrm{~g}$ dw for the mixtures for fish and salad, respectively. These macronutrients can function as signaling, recognition, and adhesion molecules, being involved in many important physiological functions, such as normal embryonic development, growth, host-pathogen interaction during infection, and development of diseases and metastases, among others [4]. Proteins are also present in considerable amounts ( 15.5 to $20.9 \mathrm{~g} / 100 \mathrm{~g} \mathrm{dw}$ ), being an important nutrient that provides a basis of energy and essential amino acids for the growth and maintenance of the human health, once they may have specific biological activities that prevent some diseases, particularly at a cardiovascular and bone level, while promoting weight control and satiety [23].

Table 1. Nutritional value of the condiment mixtures ( $\mathrm{g} / 100 \mathrm{~g} \mathrm{dw}$ and $\mathrm{kcal} / 100 \mathrm{~g} \mathrm{dw}$ for energy; mean $\pm S D)$.

\begin{tabular}{ccccc}
\hline & $\begin{array}{c}\text { Mixture for } \\
\text { Poultry }\end{array}$ & $\begin{array}{c}\text { Mixture for } \\
\text { Meat }\end{array}$ & $\begin{array}{c}\text { Mixture for } \\
\text { Salad }\end{array}$ & Mixture for Fish \\
\hline Proteins & $18.1 \pm 0.2^{\mathrm{b}}$ & $16.82 \pm 0.07^{\mathrm{c}}$ & $15.5 \pm 0.2^{\mathrm{d}}$ & $20.9 \pm 0.3^{\mathrm{a}}$ \\
Ashes & $9.9 \pm 0.1^{\mathrm{b}}$ & $8.9 \pm 0.2^{\mathrm{d}}$ & $9.20 \pm 0.04^{\mathrm{c}}$ & $10.8 \pm 0.3^{\mathrm{a}}$ \\
Fat & $5.5 \pm 0.1^{\mathrm{a}}$ & $3.81 \pm 0.03^{\mathrm{b}}$ & $3.294 \pm 0.006^{\mathrm{c}}$ & $3.02 \pm 0.02^{\mathrm{d}}$ \\
Carbohydrates & $66.52 \pm 0.04^{\mathrm{c}}$ & $70.5 \pm 0.2^{\mathrm{b}}$ & $72.0 \pm 0.1^{\mathrm{a}}$ & $65.2 \pm 0.6^{\mathrm{d}}$ \\
Energy & $387.534 \pm 0.001^{\mathrm{a}}$ & $383.3 \pm 0.8^{\mathrm{b}}$ & $379.7 \pm 0.2^{\mathrm{c}}$ & $372 \pm 1^{\mathrm{d}}$ \\
\hline
\end{tabular}

$\bar{a}, \mathrm{~b}, \mathrm{c}, \mathrm{d}$ : In each line, different letters represent statistically significant differences $(p<0.05)$.

\subsection{Chemical Composition}

\subsubsection{Free Sugars}

Free sugars were also analyzed, and the results are presented in Table 2. Fructose, sucrose, and glucose were detected in all mixtures, with that for salad showing the highest total sugars $(8.4 \mathrm{~g} / 100 \mathrm{~g} \mathrm{dw})$ and the mixture for meat the slightest $(4.28 \mathrm{~g} / 100 \mathrm{~g} \mathrm{dw})$. In a previous study [4] where several condiments were analyzed, A. schoenoprasum was found to be the plant with the highest fructose content, which could explain the low amount detected in the mixture for meat, once this plant is not present in its constitution. Also, the same authors found that $T$. citriodorus, present in the seasoning mixture for fish, holds high amounts of sucrose, thus influencing its greater values. The small concentration of sugars is part of a minimal share of carbohydrates, making these condiments suitable for a low-calorie, balanced, and diversified diet.

Table 2. Free sugars composition of the condiment mixtures (mg/100 $\mathrm{g} \mathrm{dw}$; mean $\pm \mathrm{SD}$ ).

\begin{tabular}{ccccc}
\hline & $\begin{array}{c}\text { Mixture for } \\
\text { Poultry }\end{array}$ & $\begin{array}{c}\text { Mixture for } \\
\text { Meat }\end{array}$ & $\begin{array}{c}\text { Mixture for } \\
\text { Salad }\end{array}$ & Mixture for Fish \\
\hline Fructose & $3.0 \pm 0.1^{\mathrm{b}}$ & $0.86 \pm 0.03^{\mathrm{d}}$ & $3.4 \pm 0.2^{\mathrm{a}}$ & $2.26 \pm 0.05^{\mathrm{c}}$ \\
Glucose & $2.2 \pm 0.1^{\mathrm{b}}$ & $1.03 \pm 0.03^{\mathrm{d}}$ & $2.7 \pm 0.1^{\mathrm{a}}$ & $1.61 \pm 0.01^{\mathrm{c}}$ \\
Sucrose & $1.75 \pm 0.04^{\mathrm{d}}$ & $2.38 \pm 0.03^{\mathrm{b}}$ & $2.27 \pm 0.03^{\mathrm{c}}$ & $2.56 \pm 0.04^{\mathrm{a}}$ \\
Total & $7.0 \pm 0.2^{\mathrm{b}}$ & $4.28 \pm 0.04^{\mathrm{d}}$ & $8.4 \pm 0.3^{\mathrm{a}}$ & $6.427 \pm 0.004^{\mathrm{c}}$ \\
\hline
\end{tabular}

$\overline{a, b, c, d}$ : In each line, different letters represent statistically significant differences $(p<0.05)$. Equations of the calibration lines obtained with commercial standards: fructose $\left(y=1.04 x, R^{2}=0.999 ; \mathrm{LOD}=0.05 \mathrm{mg} / \mathrm{mL}\right.$; $\mathrm{LOQ}=0.18 \mathrm{mg} / \mathrm{mL})$; glucose $\left(\mathrm{y}=0.935 \mathrm{x}, \mathrm{R}^{2}=0.999 ; \mathrm{LOD}=0.08 \mathrm{mg} / \mathrm{mL} ; \mathrm{LOQ}=0.25 \mathrm{mg} / \mathrm{mL}\right) ;$ and sucrose $\left(\mathrm{y}=1.17675 \mathrm{x}, \mathrm{R}^{2}=0.997 ; \mathrm{LOD}=0.06 \mathrm{mg} / \mathrm{mL} ; \mathrm{LOQ}=0.30 \mathrm{mg} / \mathrm{mL}\right)$.

\subsubsection{Organic Acids}

The results achieved for organic acids are shown in Table 3, with the mixture for meat presenting the highest total concentration $(4.17 \mathrm{mg} / 100 \mathrm{~g} \mathrm{dw})$, followed by those for poultry, fish, and salad $(3.65,3.6$, and $2.63 \mathrm{mg} / 100 \mathrm{~g} \mathrm{dw}$, respectively). Malic acid was 
found to be the most abundant organic acid in all samples, with higher concentration in the mixture for meat $(2.34 \mathrm{mg} / 100 \mathrm{~g})$. Once P. crispum is present in all the studied mixtures, and given the fact that malic acid is also prevalent in this species, its higher concentration in the mixtures may be related to its presence [24]. Also, T. mastichina, present in the mixture for meat, presents malic acid in large amounts, which may also contribute to its greater evidence in this sample. On the other hand, citric acid showed the lowest values in the mixtures of poultry and meat $(0.95$ and $0.88 \mathrm{mg} / 100 \mathrm{~g} \mathrm{dw}$, respectively), not being detected in the mixture for salad. Also, fumaric acid was only detected in trace amounts in the mixtures for poultry and meat. Thus, in addition to the ability of preventing oxidative stress, these extracts could also be included in food formulations as acidulants, given the presence of citric and malic acids in their composition [25]. These compounds have also been reported to be responsible for cardioprotective effects, in which adjacent mechanisms may be related to their anti-inflammatory and antiplatelet effects [26]. Other authors reported that malic acid, the major organic acid present in all of the studied mixtures, has good antioxidant properties and is able to reduce cell apoptosis [27]. It also demonstrated antimicrobial activity against Listeria monocytogenes, Salmonella Enteritidis, and Escherichia coli O157:H7. On the other hand, fumaric acid derivatives have shown effectiveness against psoriasis and in the prevention of cardiovascular and diabetic diseases, as well as citric acid, which can also improve ketosis and protect against the development of diabetic complications [28]. In addition, these compounds act as precursors of phenolic and flavoring compounds [29].

Table 3. Organic acids composition of the condiment mixtures (mg/100 g dw; mean \pm SD).

\begin{tabular}{ccccc}
\hline & $\begin{array}{c}\text { Mixture for } \\
\text { Poultry }\end{array}$ & $\begin{array}{c}\text { Mixture for } \\
\text { Meat }\end{array}$ & $\begin{array}{c}\text { Mixture for } \\
\text { Salad }\end{array}$ & Mixture for Fish \\
\hline Oxalic acid & $1.32 \pm 0.01^{\mathrm{a}}$ & $0.94 \pm 0.02^{\mathrm{b}}$ & $0.78 \pm 0.03^{\mathrm{c}}$ & $0.08 \pm 0.01^{\mathrm{d}}$ \\
Malic acid & $1.38 \pm 0.04^{\mathrm{d}}$ & $2.34 \pm 0.04^{\mathrm{a}}$ & $1.85 \pm 0.04^{\mathrm{b}}$ & $1.64 \pm 0.08^{\mathrm{c}}$ \\
Citric acid & $0.95 \pm 0.03^{\mathrm{b}}$ & $0.88 \pm 0.02^{\mathrm{b}}$ & nd & $1.25 \pm 0.07^{\mathrm{a}}$ \\
Fumaric acid & $\operatorname{tr}^{\text {Tr }}$ & $\operatorname{tr}^{\mathrm{a}}$ & nd & nd $^{\mathrm{b}}$ \\
Total & $3.65 \pm 0.09^{\mathrm{b}}$ & $4.17 \pm 0.03^{\mathrm{a}}$ & $2.63 \pm 0.07^{\mathrm{c}}$ & $3.6 \pm 0.1^{\mathrm{b}}$ \\
\hline
\end{tabular}

tr: traces; nd: not detected. ${ }^{a}, b, c, d$ : In each line, different letters represent statistically significant differences $(p<0.05)$. Equations of the calibration lines obtained with commercial standards: oxalic acid $\left(\mathrm{y}=1 \times 10^{7} \mathrm{x}+23,1891 ; \mathrm{R}^{2}=0.9999 ; \quad \mathrm{LOD}=12.55 \mu \mathrm{g} / \mathrm{mL} ; \mathrm{LOQ}=41.82 \mu \mathrm{g} / \mathrm{mL}\right) ;$ malic acid $\left(y=950,041 x+6255.6 ; R^{2}=0.9999 ; \mathrm{LOD}=36 \mu \mathrm{g} / \mathrm{mL} ; \mathrm{LOQ}=120 \mu \mathrm{g} / \mathrm{mL}\right) ;$ citric acid $\left(\mathrm{y}=1 \times 10^{6} \mathrm{x}-10,277 ;\right.$ $\left.\mathrm{R}^{2}=0.9997 ; \mathrm{LOD}=0.11 \mu \mathrm{g} / \mathrm{mL} ; \mathrm{LOQ}=0.34 \mu \mathrm{g} / \mathrm{mL}\right) ;$ and fumaric acid $\left(\mathrm{y}=1 \times 10^{8} \mathrm{x}+614,399 ; \mathrm{R}^{2}=0.9986 ;\right.$ $\mathrm{LOD}=0.08 \mu \mathrm{g} / \mathrm{mL} ; \mathrm{LOQ}=0.26 \mu \mathrm{g} / \mathrm{mL})$.

\subsubsection{Tocopherols}

Regarding tocopherols, the analysis allowed the identification of the four isoforms ( $\alpha$-tocopherol, $\beta$-tocopherol, $\gamma$-tocopherol, and $\delta$-tocopherol) in all of the analyzed extracts (Table 4), with the mixtures for fish, meat, and salad presenting the highest total tocopherol values (17.1, 17.0, and $16.6 \mathrm{mg} / 100 \mathrm{~g} \mathrm{dw}$, respectively), and that for poultry showing a lowest amount (14.5 mg/100 g dw).

$\gamma$-tocopherol was the most abundant isoform found in all the samples in the study, with higher concentrations in the mixtures for fish and salad (14.1 and $13.85 \mathrm{mg} / 100 \mathrm{~g} \mathrm{dw}$, respectively) and the lowest in the mixture for poultry $(8.17 \mathrm{mg} / 100 \mathrm{~g} \mathrm{dw})$. In its turn, $\delta$-tocopherol was the least predominant isoform, with concentration values ranging from $0.80 \mathrm{mg} / 100 \mathrm{~g} \mathrm{dw}$ for the mixture for poultry to $0.134 \mathrm{mg} / 100 \mathrm{~g} \mathrm{dw}$ for the mixture for seasoning meat. The mixture for salad is composed of $\mathrm{O}$. vulgare and T. mastichina, characteristic for their composition in tocopherols, namely $\alpha$-tocopherol and $\gamma$-tocopherol, in considerable amounts [30], which may explain the high incidence of $\gamma$-tocopherol in this mixture. However, the same is not observed for $\alpha$-tocopherol, which may be due to the different concentrations of these and other plants used in the mixture composition. The mixture for meat, which also has T. mastichina and R. officinalis in its composition, 
rich in $\gamma$-tocopherol, as previously mentioned [31], may be responsible for the values presented, together with other species present in the mixture. In addition to the radical scavenging action of tocopherols, non-antioxidant functions have also been shown by these compounds, both in vivo and in vitro [32,33].

Table 4. Composition of tocopherols of the condiment mixtures (mg/100 g dw; mean \pm SD).

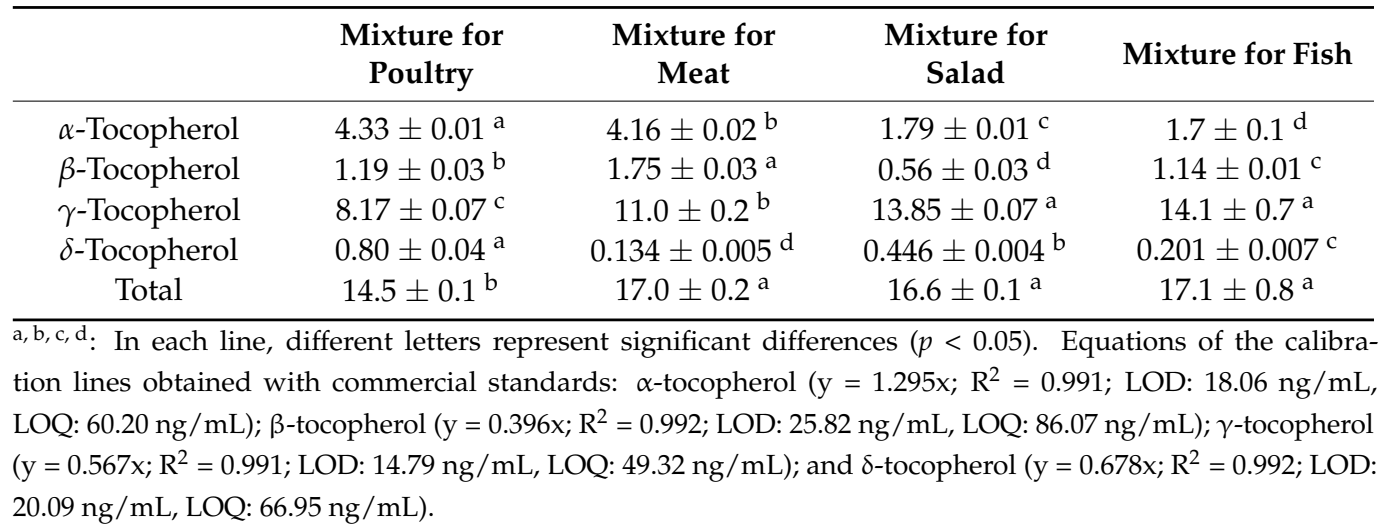

\subsubsection{Fatty Acids}

The lipid fraction in MAPs is generally low and data on the composition of fatty acids is essential, especially with respect to the content of polyunsaturated fatty acids (PUFA) and saturated fatty acids (SFA) [34]. The fatty acid composition of the mixtures under study is shown in Table 5 .

To the best of our knowledge, there is no available data allowing the comparison of the results obtained herein with those reported by other authors. A total of 23 different fatty acids were identified, which reflect a good fatty acids profile and corroborates the importance of the inclusion of these condiments in a balanced diet. Additionally, it is worth highlighting the prevalence of PUFA (between 57 and 65.8\%) compared to SFA (between 30.5 and 39\%). In all mixtures, the SFAs found in greater quantity were palmitic (C16:0) and stearic acid (C18:0), whereas the mostly present MUFA (monounsaturated fatty acids) were palmitoleic $(\mathrm{C} 16: 1)$ and oleic $(\mathrm{C} 18: 1 \mathrm{n} 9 \mathrm{c})$ acids. Finally, the most prevalent PUFAs were linoleic acid (C18:2n6c), in values ranging from 18.6 to $21.47 \%$, and $\alpha$-linolenic acid (C18:3n3; 36 to $42.7 \%$ ). Given the important role of linoleic and $\alpha$-linolenic acids as precursors of omega- 6 and omega- 3 fatty acids, and the fact that these compounds cannot be synthesized in the human organism and must be obtained from the diet [4], it is of great interest to verify its presence in the studied mixtures.

Table 5. Fatty acids composition of the condiment mixtures (relative \%; mean $\pm \mathrm{SD}$ ).

\begin{tabular}{ccccc}
\hline & $\begin{array}{c}\text { Mixture for } \\
\text { Poultry }\end{array}$ & $\begin{array}{c}\text { Mixture for } \\
\text { Meat }\end{array}$ & $\begin{array}{c}\text { Mixture for } \\
\text { Salad }\end{array}$ & Mixture for Fish \\
\hline C6:0 & $0.1725 \pm 0.0007$ & $0.808 \pm 0.008$ & nd & $0.75 \pm 0.04$ \\
C11:0 & $1.00 \pm 0.02$ & $1.221 \pm 0.005$ & $1.071 \pm 0.014$ & $0.733 \pm 0.007$ \\
C12:0 & $0.21 \pm 0.01$ & $0.142 \pm 0.001$ & $0.70 \pm 0.02$ & $0.138 \pm 0.001$ \\
C13:0 & $1.22 \pm 0.08$ & $1.251 \pm 0.007$ & $1.03 \pm 0.02$ & $1.17 \pm 0.05$ \\
C14:0 & $1.128 \pm 0.003$ & $1.01 \pm 0.03$ & $1.57 \pm 0.02$ & $0.81 \pm 0.02$ \\
C15:0 & $0.816 \pm 0.006$ & $0.97 \pm 0.01$ & $0.297 \pm 0.009$ & $0.211 \pm 0.004$ \\
C16:0 & $15.7 \pm 0.6$ & $18.51 \pm 0.03$ & $18.6 \pm 0.9$ & $13.8 \pm 0.2$ \\
C16:1 & $1.24 \pm 0.02$ & $0.99 \pm 0.01$ & $0.810 \pm 0.006$ & $1.041 \pm 0.008$ \\
C17:0 & $1.060 \pm 0.007$ & $1.53 \pm 0.04$ & $0.762 \pm 0.003$ & $0.96 \pm 0.06$ \\
C18:0 & $4.1 \pm 0.1$ & $4.67 \pm 0.06$ & $6.33 \pm 0.02$ & $4.0 \pm 0.1$ \\
\hline
\end{tabular}


Table 5. Cont.

\begin{tabular}{ccccc}
\hline & $\begin{array}{c}\text { Mixture for } \\
\text { Poultry }\end{array}$ & $\begin{array}{c}\text { Mixture for } \\
\text { Meat }\end{array}$ & $\begin{array}{c}\text { Mixture for } \\
\text { Salad }\end{array}$ & Mixture for Fish \\
\hline C18:1n9c & $2.006 \pm 0.01$ & $1.66 \pm 0.01$ & $2.63 \pm 0.07$ & $1.32 \pm 0.01$ \\
C18:2n6c & $21.1 \pm 0.3$ & $18.6 \pm 0.3$ & $19.8 \pm 0.2$ & $21.47 \pm 0.04$ \\
C18:3n3 & $42.7 \pm 0.2$ & $39.3 \pm 0.2$ & $36 \pm 1$ & $42.3 \pm 0.8$ \\
C20:0 & $1.189 \pm 0.002$ & $1.57 \pm 0.04$ & $2.01 \pm 0.07$ & $3.3 \pm 0.1$ \\
C20:1 & $0.163 \pm 0.009$ & $0.192 \pm 0.007$ & $0.094 \pm 0.003$ & $0.081 \pm 0.004$ \\
C20:2 & $0.106 \pm 0.004$ & $0.086 \pm 0.001$ & $0.067 \pm 0.001$ & $0.126 \pm 0.006$ \\
C21:0 & $0.106 \pm 0.007$ & $0.244 \pm 0.002$ & $0.182 \pm 0.001$ & $0.122 \pm 0.003$ \\
C20:4n6 & $1.215 \pm 0.003$ & $1.65 \pm 0.01$ & nd & $0.50 \pm 0.01$ \\
C22:0 & $1.50 \pm 0.05$ & $1.818 \pm 0.005$ & $3.314 \pm 0.005$ & $2.72 \pm 0.01$ \\
C20:5n3 & $0.253 \pm 0.005$ & $0.53 \pm 0.03$ & nd & $0.332 \pm 0.004$ \\
C22:2 & $0.62 \pm 0.03$ & $0.78 \pm 0.04$ & $0.58 \pm 0.02$ & $0.86 \pm 0.05$ \\
C24:0 & $2.279 \pm 0.009$ & $2.109 \pm 0.006$ & $3.39 \pm 0.04$ & $3.259 \pm 0.002$ \\
C24:1 & $0.1805 \pm 0.0007$ & $0.363 \pm 0.006$ & $0.334 \pm 0.006$ & $0.041 \pm 0.001$ \\
SFA & $30.5 \pm 0.5^{\mathrm{a}}$ & $35.85 \pm 0.09^{\mathrm{b}}$ & $39 \pm 1^{\mathrm{c}}$ & $31.9 \pm 0.7^{\mathrm{d}}$ \\
MUFA & $3.59 \pm 0.01^{\mathrm{a}}$ & $3.21 \pm 0.02^{\mathrm{c}}$ & $3.87 \pm 0.06^{\mathrm{b}}$ & $2.478 \pm 0.008^{\mathrm{d}}$ \\
PUFA & $65.8 \pm 0.5^{\mathrm{a}}$ & $60.9 \pm 0.1^{\mathrm{b}}$ & $57 \pm 1^{\mathrm{d}}$ & $65.5 \pm 0.7^{\mathrm{c}}$ \\
\hline
\end{tabular}

$\overline{a, b, c, d}$ : In each line, different letters represent statistically significant differences $(p<0.05)$. Caproic acid (C6:0); undecylic acid (C11:0); lauric acid (C12:0); tridecanoic acid (C13:0); myristic acid (C14:0); pentadecylic acid (C15:0); palmitic acid (C16:0); palmitoleic acid (C16:1); margaric acid (C17:0); stearic acid (C18:0); oleic acid (C18:1n9c); linoleic acid (C18:2n6c); $\alpha$-linolenic acid (C18:3n3); arachidic acid (C20:0); cis-11-eicosenoic acid (C20:1); cis11,14-eicosadienoic acid (C20:2); heneicosylic acid (C21:0); arachidonic acid (C20:4n6); behenic acid (C22:0); cis-5,8,11,14,17-eicosapentaenoic acid (C20:5n3); cis-13,16-docosadienoic acid (C22:2); lignoceric acid (C24:0); nervonic acid (C24:1); SFA: saturated fatty acids; MUFA: monounsaturated fatty acids; PUFA: polyunsaturated fatty acids.

\subsubsection{Phenolic Compounds}

The tentative identification of the phenolic compounds found in the four condiment mixtures, as well as the retention times (Rt), maximum absorbance $\left(\lambda_{\max }\right)$, pseudomolecular ion $\left([\mathrm{M}-\mathrm{H}]^{-}\right)$, and the main ion fragments $\left(\mathrm{MS}^{2}\right)$ of each phenolic compound are presented in Table 6. The attempt to identify the individual phenolic compounds was based on the data presented and, whenever possible, in comparison to the available standard compounds and/or with the existing literature. Among the twenty-five compounds detected and tentatively identified, thirteen were phenolic acids and eleven were flavonoids. Peaks $5,10,14,15$, and 16 were tentatively identified by comparing their retention time and UV spectrum with available standards, as caffeic acid, quercetin-3-O-glucoside, luteolin-7-Oglucoside, and cis and trans rosmarinic acid, respectively. Particularly, for peaks 15 and 16, the standard compound presented the same retention time of peak 16, identified as the trans isoform, and peak $\mathbf{1 5}$ (with the same chromatographic response as the previous peak) as the cis isoform. Regarding phenolic acids, peaks 2 ([M-H $]^{-}$at $\left.m / z 353\right), 3\left([\mathrm{M}-\mathrm{H}]^{-}\right.$ at $m / z 341), 6 / 7\left([\mathrm{M}-\mathrm{H}]^{-}\right.$at $\left.m / z 325\right), 11\left([\mathrm{M}-\mathrm{H}]^{-}\right.$at $\left.m / z 421\right), 12\left([\mathrm{M}-\mathrm{H}]^{-}\right.$at $\left.m / z 521\right), 17$ ([M-H] $]^{-}$at $\left.m / z 717\right), 19\left([\mathrm{M}-\mathrm{H}]^{-}\right.$at $\left.m / z 537\right), 23\left([\mathrm{M}-\mathrm{H}]^{-}\right.$at $\left.m / z 493\right)$ and 24/25 $\left([\mathrm{M}-\mathrm{H}]^{-}\right.$at $m / z 557)$ were tentatively identified by comparing their chromatographic responses with the previously described $[35,36]$, being therefore tentatively identified as 3-O-caffeoylquinic acid, caffeic acid hexoside [37], p-coumaric acid hexoside [37], 4-Hydroxy-7-O-(3-hydroxy4-O-glucosylbenzoyl)benzyl alcohol [38], rosmarinic acid hexoside [39], salvianolic acid B [39], lithospermic acid A, salvianolic acid A [39], and p-coumaric acid isomers I/II [40], respectively. The group of the flavonoid compounds, peaks $4\left([\mathrm{M}-\mathrm{H}]^{-}\right.$at $\left.\mathrm{m} / \mathrm{z} 593\right)$, $9\left([\mathrm{M}-\mathrm{H}]^{-}\right.$at $\left.m / z 1093\right)$, and $13\left([\mathrm{M}-\mathrm{H}]^{-}\right.$at $\left.m / z 461\right)$ were also tentatively identified by comparing their chromatographic response with the previously described in the literature, being therefore identified as apigenin- $C$-dihexoside [41], kaempferol- $O$-glycosyl-( $p$-coumaroylhexosyl)-hexosyl-glucuronide [42], and luteolin-7-O-glucuronide [37]. The remaining peaks belonging to the group of flavonoids were tentatively identified by analyzing their pseudomolecular ion and $\mathrm{MS}^{2}$ fragments. Peak 8 presents a pseudomolecular ion $[\mathrm{M}-\mathrm{H}]^{-}$at $m / z$ 
725 and fragments MS ${ }^{2}$ at $m / z 681(44 \mathrm{u}), 519(162 \mathrm{u}), 357(162 \mathrm{u}), 315(42 \mathrm{u}$, isorhamnetin aglycone), which corresponds to the loss of a malonyl group and two hexosides, being tentatively identified as isorhamnetin-O-malonyl-dihexoside. Peak $18\left([\mathrm{M}-\mathrm{H}]^{-}\right.$at $\mathrm{m} / z$ 593) showed a single MS ${ }^{2}$ fragment at $m / z 285$ (luteolin aglycone, $308 \mathrm{u}$ ), and was tentatively identified as luteolin-7-O-rutinoside. Peaks 20 and 21, present a very similar chromatographic response, with a pseudomolecular ion $[\mathrm{M}-\mathrm{H}]^{-}$at $m / z 649$ and with $\mathrm{MS}^{2}$ fragments at $m / z 605$ and $563(44+42 \mathrm{u}$, malonyl group), and $269(132+162 \mathrm{u}$, pentosyl and hexosyl groups) being therefore tentatively identified as apigenin-O-malonyl-pentosyl-hexoside. Peak 22, shows a pseudomolecular ion $[\mathrm{M}-\mathrm{H}]^{-}$at $m / z 679$ with $\mathrm{MS}^{2}$ fragments at $m / z 635$ and $593(44+42 \mathrm{u}$, malonyl group) and $299(132+162 \mathrm{u}$, pentosyl and hexosyl groups), being tentatively identified as diosmetin-O-malonyl-hexosyl-pentoside. Finally, one flavan3-ol was identified (peak 1), with a pseudomolecular ion $[\mathrm{M}-\mathrm{H}]^{-}$at $\mathrm{m} / \mathrm{z} 305$, a maximum absorption spectrum at $270 \mathrm{~nm}$ and $\mathrm{MS}^{2}$ fragments characteristic of a (epi) galocatechin, being tentatively identified based on the previously described by Bouziane et al. [43]

Table 6. Retention times (Rt), wavelengths of maximum absorption in the visible region $\left(\lambda_{\max }\right)$, mass spectral data, and tentative identification of the phenolic compounds present in the condiment mixtures hydroethanolic extracts.

\begin{tabular}{|c|c|c|c|c|c|c|}
\hline Peak & Rt (min) & $\lambda_{\max }(\mathrm{nm})$ & $\begin{array}{c}{[\mathrm{M}-\mathrm{H}]^{-}} \\
m / z\end{array}$ & $\mathrm{MS}^{2} m / z$ & $\begin{array}{c}\text { Attempted } \\
\text { Identification }\end{array}$ & $\begin{array}{l}\text { Reference/Method } \\
\text { Used for } \\
\text { Identification }\end{array}$ \\
\hline 1 & 4.95 & 270 & 305 & $219(14), 179(30), 125(100)$ & (Epi)gallocatechin & [43] \\
\hline 2 & 5.91 & 323 & 353 & 191(100),179(34),173(5),165(5) & $\begin{array}{c}3-\mathrm{O}- \\
\text { Caffeoylquinic acid }\end{array}$ & {$[35,36]$} \\
\hline 3 & 7.86 & 322 & 341 & $179(100), 135(23)$ & $\begin{array}{l}\text { Caffeic acid } \\
\text { hexoside }\end{array}$ & [37] \\
\hline 4 & 8.9 & 325 & 593 & $\begin{array}{c}575(12), 503(35), 473(100), 383(14), \\
353(21)\end{array}$ & $\begin{array}{l}\text { Apigenin-C- } \\
\text { dihexoside }\end{array}$ & [41] \\
\hline 5 & 9.37 & 322 & 179 & $135(100)$ & Caffeic acid & $\begin{array}{l}\text { Standard } \\
\text { compound }\end{array}$ \\
\hline 6 & 10.3 & 280 & 325 & $163(100), 119(25)$ & $\begin{array}{l}p \text {-Coumaric acid } \\
\text { hexoside }\end{array}$ & [37] \\
\hline 7 & 11.36 & 284 & 325 & $163(100), 119(25)$ & $\begin{array}{l}p \text {-Coumaric acid } \\
\text { hexoside }\end{array}$ & [37] \\
\hline 8 & 12.82 & 326 & 725 & $681(42), 519(100), 357(23), 315(67)$ & $\begin{array}{c}\text { Isorhamnetin-O- } \\
\text { malonyl- } \\
\text { dihexoside }\end{array}$ & DAD/MS \\
\hline 9 & 13.84 & 320 & 1093 & $917(100), 285(13)$ & $\begin{array}{l}\text { Kaempherol-O- } \\
\text { glycosyl- }(p- \\
\text { coumaroyl- } \\
\text { hexosyl)hexosyl- } \\
\text { glucoronide }\end{array}$ & [42] \\
\hline 10 & 15.05 & 343 & 463 & $301(100)$ & $\begin{array}{l}\text { Quercetin-3-O- } \\
\text { glucoside } \\
\text { 4-Hydroxy-7-O-(3- }\end{array}$ & $\begin{array}{l}\text { Standard } \\
\text { compound }\end{array}$ \\
\hline 11 & 15.96 & $263 / 294 / 336$ & 421 & $259(54), 153(100), 108(10)$ & $\begin{array}{l}\text { hydroxy-4-O- } \\
\text { glucosylbenzoyl) } \\
\text { benzyl alcohol }\end{array}$ & [38] \\
\hline 12 & 16.87 & 346 & 521 & $359(100), 197(35), 179(40), 161(98)$ & $\begin{array}{l}\text { Rosmarinic acid } \\
\text { hexoside }\end{array}$ & [39] \\
\hline
\end{tabular}


Table 6. Cont.

\begin{tabular}{|c|c|c|c|c|c|c|}
\hline Peak & Rt (min) & $\lambda_{\max }(\mathrm{nm})$ & $\begin{array}{l}{[\mathrm{M}-\mathrm{H}]^{-}} \\
\quad m / z\end{array}$ & $\mathrm{MS}^{2} m / z$ & $\begin{array}{c}\text { Attempted } \\
\text { Identification }\end{array}$ & $\begin{array}{l}\text { Reference/Method } \\
\text { Used for } \\
\text { Identification }\end{array}$ \\
\hline 13 & 17.69 & 345 & 461 & 285(100) & $\begin{array}{l}\text { Luteolin-7-O- } \\
\text { glucuronide }\end{array}$ & [37] \\
\hline 14 & 18.01 & 344 & 447 & $285(100)$ & $\begin{array}{l}\text { Luteolin-7-O- } \\
\text { glucoside }\end{array}$ & $\begin{array}{l}\text { Standard } \\
\text { compound }\end{array}$ \\
\hline 15 & 20.64 & 328 & 359 & 197(35),179(39),161(100) & cis Rosmarinic acid & $\begin{array}{l}\text { Standard } \\
\text { compound }\end{array}$ \\
\hline 16 & 21.04 & 330 & 359 & 197(35),179(39),161(100) & $\begin{array}{l}\text { trans Rosmarinic } \\
\text { acid }\end{array}$ & $\begin{array}{l}\text { Standard } \\
\text { compound }\end{array}$ \\
\hline 17 & 22.41 & 334 & 717 & $537(5), 519(100), 475(13), 339(31)$ & Salvianolic acid B & [39] \\
\hline 18 & 23.5 & 337 & 593 & $285(100)$ & $\begin{array}{l}\text { Luteolin-7-O- } \\
\text { rutinoside }\end{array}$ & $\mathrm{DAD} / \mathrm{MS}$ \\
\hline 19 & 24.28 & 329 & 537 & $\begin{array}{c}493(100), 359(62), 313(15), 295(<5) \\
269(<5), 197(5), 179(5)\end{array}$ & $\begin{array}{l}\text { Lithospermic acid } \\
\text { A }\end{array}$ & [39] \\
\hline 20 & 26.03 & 336 & 649 & $605(34), 563(41), 269(100)$ & $\begin{array}{l}\text { Apigenin-O- } \\
\text { malonyl-pentosyl- } \\
\text { hexoside }\end{array}$ & $\mathrm{DAD} / \mathrm{MS}$ \\
\hline 21 & 26.97 & 339 & 649 & $605(56), 563(31), 269(100)$ & $\begin{array}{l}\text { Apigenin-O- } \\
\text { malonyl-pentosyl- } \\
\text { hexoside }\end{array}$ & $\mathrm{DAD} / \mathrm{MS}$ \\
\hline 22 & 27.67 & 340 & 679 & 635(100),593(5),299(51) & $\begin{array}{l}\text { Diosmetin-O- } \\
\text { malonyl-hexosyl- } \\
\text { pentoside }\end{array}$ & $\mathrm{DAD} / \mathrm{MS}$ \\
\hline 23 & 28.26 & 323 & 493 & $\begin{array}{c}359(100), 313(12), 295(5) \\
197(10), 179(10)\end{array}$ & Salvianolic acid A & [39] \\
\hline 24 & 31.21 & $287 / 313$ & 557 & $513(5), 469(37), 349(100), 163(34)$ & $\begin{array}{c}p \text {-Coumaric acid } \\
\text { derivative isomer I }\end{array}$ & [40] \\
\hline 25 & 31.66 & $290 / 310$ & 557 & $513(5), 469(37), 349(100), 163(34)$ & $\begin{array}{c}p \text {-Coumaric acid } \\
\text { derivative isomer II }\end{array}$ & [40] \\
\hline
\end{tabular}

The quantification data of the phenolic compounds present in the analyzed extracts are shown in Table 7. The mixture for meat showed the highest concentration in phenolic compounds $(101.2 \mathrm{mg} / \mathrm{g})$, followed by those for fish $(76.1 \mathrm{mg} / \mathrm{g})$ and for poultry $(68.2 \mathrm{mg} / \mathrm{g})$, with the extract of the mixture for salad presenting the lowest content in phenolic compounds $(52.1 \mathrm{mg} / \mathrm{g})$. In all extracts, the most abundant compound is apigenin-O-malonyl-pentosyl-hexoside, which may be due, once again, to the presence of P. crispum in all of the mixtures, since it is a predominant aglycone in this plant, as well as luteolin, quercetin and isorhamnetin, also present in considerable amounts [44,45]. Regarding phenolic acids, the most prevalent was rosmarinic acid (cis and trans isoforms), being more abundant in mixtures for meat and poultry.

Table 7. Quantification (mg/g of extract) of the phenolic compounds present in the condiment mixtures hydroethanolic extracts (mean $\pm \mathrm{SD}$ ).

\begin{tabular}{|c|c|c|c|c|}
\hline Peak & $\begin{array}{l}\text { Mixture for } \\
\text { Poultry }\end{array}$ & $\begin{array}{l}\text { Mixture for } \\
\text { Meat }\end{array}$ & $\begin{array}{l}\text { Mixture for } \\
\text { Salad }\end{array}$ & Mixture for Fish \\
\hline 1 & $0.57 \pm 0.01^{\mathrm{c}}$ & $1.11 \pm 0.05^{\mathrm{a}}$ & $0.42 \pm 0.01^{\mathrm{d}}$ & $0.76 \pm 0.03^{b}$ \\
\hline 2 & $1.31 \pm 0.01^{\mathrm{c}}$ & $1.46 \pm 0.06^{\mathrm{b}}$ & $1.05 \pm 0.07^{\mathrm{d}}$ & $2.2 \pm 0.1^{\mathrm{a}}$ \\
\hline 3 & $0.21 \pm 0.01$ & $\operatorname{tr}$ & $\operatorname{tr}$ & $0.162 \pm 0.002$ \\
\hline 4 & $0.63 \pm 0.03^{c}$ & $0.752 \pm 0.002^{\mathrm{a}}$ & $0.36 \pm 0.01^{\mathrm{d}}$ & $0.68 \pm 0.01^{b}$ \\
\hline 5 & $0.19 \pm 0.01$ & $\operatorname{tr}$ & $0.185 \pm 0.005$ & nd \\
\hline 6 & $0.46 \pm 0.04^{\mathrm{a}}$ & $0.130 \pm 0.002^{c}$ & $0.128 \pm 0.002^{c}$ & $0.21 \pm 0.01^{b}$ \\
\hline 7 & $0.31 \pm 0.01^{\mathrm{d}}$ & $0.40 \pm 0.03^{c}$ & $0.577 \pm 0.003^{b}$ & $0.87 \pm 0.02^{\mathrm{a}}$ \\
\hline
\end{tabular}


Table 7. Cont.

\begin{tabular}{ccccc}
\hline Peak & $\begin{array}{c}\text { Mixture for } \\
\text { Poultry }\end{array}$ & $\begin{array}{c}\text { Mixture for } \\
\text { Meat }\end{array}$ & $\begin{array}{c}\text { Mixture for } \\
\text { Salad }\end{array}$ & Mixture for Fish \\
\hline $\mathbf{8}$ & $4.69 \pm 0.02^{\mathrm{d}}$ & $4.900 \pm 0.001^{\mathrm{a}}$ & $4.75 \pm 0.02^{\mathrm{c}}$ & $4.83 \pm 0.02^{\mathrm{b}}$ \\
$\mathbf{9}$ & $4.65 \pm 0.01^{\mathrm{b}}$ & $4.65 \pm 0.01^{\mathrm{b}}$ & $4.66 \pm 0.02^{\mathrm{b}}$ & $6.59 \pm 0.03^{\mathrm{a}}$ \\
$\mathbf{1 0}$ & nd & $7.8 \pm 0.2^{\mathrm{a}}$ & $6.46 \pm 0.08^{\mathrm{b}}$ & $5.475 \pm 0.001^{\mathrm{c}}$ \\
$\mathbf{1 1}$ & $4.69 \pm 0.01^{\mathrm{a}}$ & $4.69 \pm 0.03^{\mathrm{a}}$ & $4.66 \pm 0.01^{\mathrm{b}}$ & $4.69 \pm 0.01^{\mathrm{a}}$ \\
$\mathbf{1 2}$ & $0.67 \pm 0.01^{\mathrm{b}}$ & $2.9 \pm 0.2^{\mathrm{a}}$ & $0.384 \pm 0.003^{\mathrm{c}}$ & $2.94 \pm 0.02^{\mathrm{a}}$ \\
$\mathbf{1 3}$ & $4.92 \pm 0.06^{\mathrm{c}}$ & $5.38 \pm 0.04^{\mathrm{a}}$ & $5.3 \pm 0.1^{\mathrm{b}}$ & $5.39 \pm 0.08^{\mathrm{a}}$ \\
$\mathbf{1 4}$ & $4.9 \pm 0.1^{\mathrm{c}}$ & $7.35 \pm 0.07^{\mathrm{a}}$ & $4.98 \pm 0.02^{\mathrm{c}}$ & $5.62 \pm 0.04^{\mathrm{b}}$ \\
$\mathbf{1 5}$ & $6.0 \pm 0.2^{\mathrm{b}}$ & $10.7 \pm 0.6^{\mathrm{a}}$ & $1.47 \pm 0.06^{\mathrm{d}}$ & $2.17 \pm 0.05^{\mathrm{c}}$ \\
$\mathbf{1 6}$ & $6.8 \pm 0.4^{\mathrm{b}}$ & $14.6 \pm 0.5^{\mathrm{a}}$ & $2.53 \pm 0.07^{\mathrm{d}}$ & $4.2 \pm 0.1^{\mathrm{c}}$ \\
$\mathbf{1 7}$ & $0.54 \pm 0.02^{\mathrm{d}}$ & $0.58 \pm 0.03^{\mathrm{c}}$ & $0.63 \pm 0.01^{\mathrm{b}}$ & $1.36 \pm 0.04^{\mathrm{a}}$ \\
$\mathbf{1 8}$ & $4.7 \pm 0.1^{\mathrm{b}}$ & $4.79 \pm 0.05^{\mathrm{a}}$ & $4.67 \pm 0.01^{\mathrm{c}}$ & $4.75 \pm 0.01^{\mathrm{b}}$ \\
$\mathbf{1 9}$ & $0.49 \pm 0.03^{\mathrm{c}}$ & $0.85 \pm 0.02^{\mathrm{a}}$ & $0.49 \pm 0.02^{\mathrm{c}}$ & $0.61 \pm 0.03^{\mathrm{b}}$ \\
$\mathbf{2 0}$ & $17.3 \pm 0.9^{\mathrm{b}}$ & $23.5 \pm 0.9^{\mathrm{a}}$ & $6.3 \pm 0.3^{\mathrm{c}}$ & $17.6 \pm 0.3^{\mathrm{b}}$ \\
$\mathbf{2 1}$ & $0.89 \pm 0.02^{\mathrm{b}}$ & $1.4 \pm 0.1^{\mathrm{a}}$ & $0.64 \pm 0.02^{\mathrm{d}}$ & $0.85 \pm 0.04^{\mathrm{c}}$ \\
$\mathbf{2 2}$ & $1.12 \pm 0.04^{\mathrm{b}}$ & $1.22 \pm 0.02^{\mathrm{a}}$ & $0.64 \pm 0.05^{\mathrm{d}}$ & $1.064 \pm 0.002^{\mathrm{c}}$ \\
$\mathbf{2 3}$ & $0.99 \pm 0.07^{\mathrm{b}}$ & $0.76 \pm 0.06^{\mathrm{c}}$ & $0.43 \pm 0.01^{\mathrm{d}}$ & $2.1 \pm 0.1^{\mathrm{a}}$ \\
$\mathbf{2 4}$ & $0.427 \pm 0.002^{\mathrm{b}}$ & $0.53 \pm 0.03^{\mathrm{a}}$ & $0.16 \pm 0.01^{\mathrm{d}}$ & $0.43 \pm 0.02^{\mathrm{c}}$ \\
$\mathbf{2 5}$ & $0.516 \pm 0.004^{\mathrm{c}}$ & $0.738 \pm 0.003^{\mathrm{a}}$ & $0.25 \pm 0.01^{\mathrm{d}}$ & $0.56 \pm 0.02^{\mathrm{b}}$ \\
\hline Total Flavonoids & $45 \pm 1^{\mathrm{c}}$ & $61.8 \pm 0.9^{\mathrm{a}}$ & $39.1 \pm 0.6^{\mathrm{d}}$ & $53.6 \pm 0.6^{\mathrm{b}}$ \\
\hline Acids & $23.6 \pm 0.7^{\mathrm{b}}$ & $39.4 \pm 0.4^{\mathrm{a}}$ & $12.9 \pm 0.2^{\mathrm{d}}$ & $22.46 \pm 0.04^{\mathrm{c}}$ \\
\hline Total & $68 \pm 2^{\mathrm{c}}$ & $101.2 \pm 0.6^{\mathrm{a}}$ & $52.1 \pm 0.8^{\mathrm{d}}$ & $76.1 \pm 0.6^{\mathrm{b}}$ \\
\hline
\end{tabular}

tr: traces; nd: not detected; Calibration curves used: chlorogenic acid ( $\mathrm{y}=168823 \mathrm{x}-161172 ; \mathrm{R}^{2}=0.9999, \mathrm{LOD}$ (Limit of detection) $=0.20 \mu \mathrm{g} / \mathrm{mL}$ and LOQ (Limit of quantification) $=0.68 \mu \mathrm{g} / \mathrm{mL}$ peak 2); $p$-coumaric acid $\left(\mathrm{y}=301,950 \mathrm{x}+6966.7, \mathrm{R}^{2}=0.9999, \mathrm{LOD}=0.68 \mu \mathrm{g} / \mathrm{mL} ; \mathrm{LOQ}=1.61 \mu \mathrm{g} / \mathrm{mL}\right.$, peaks 6, 7, 24 and 25); rosmarinic acid $\left(\mathrm{y}=191291 \mathrm{x}-652,903, \mathrm{R}^{2}=0.999, \mathrm{LOD}=0.15 \mu \mathrm{g} / \mathrm{mL} ; \mathrm{LOQ}=0.68 \mu \mathrm{g} / \mathrm{mL}\right.$ peaks $12,15,16,17,19$ and 23); caffeic acid ( $\mathrm{y}=388,345 \mathrm{x}+406,369, \mathrm{R}^{2}=0.99 ; \mathrm{LOD}=0.78 \mu \mathrm{g} / \mathrm{mL} ; \mathrm{LOQ}=1.97 \mu \mathrm{g} / \mathrm{mL}$, peaks 3 and 5); apigenin-6-C-glucoside ( $\mathrm{y}=107,025 \mathrm{x}+61,531, \mathrm{R}^{2}=0.998 ; \mathrm{LOD}=0.19 \mu \mathrm{g} / \mathrm{mL} ; \mathrm{LOQ}=0.63 \mu \mathrm{g} / \mathrm{mL}$ peak 4$)$; apigenin-7-O-glucoside $\left(\mathrm{y}=10,683 \mathrm{x}-45,794, \mathrm{R}^{2}=0.996, \mathrm{LOD}=0.10 \mu \mathrm{g} / \mathrm{mL} ; \mathrm{LOQ}=0.53 \mu \mathrm{g} / \mathrm{mL}\right.$, peaks 20,21 and 22); (+)-catechin $\left(\mathrm{y}=84,950 \mathrm{x}-23,200, \mathrm{R}^{2}=1, \mathrm{LOD}=0.17 \mu \mathrm{g} / \mathrm{mL} ; \mathrm{LOQ}=0.68 \mu \mathrm{g} / \mathrm{mL}\right.$, peak 1); quercetin-3O-glucoside $\left(\mathrm{y}=34,843 \mathrm{x}-16,0173, \mathrm{R}^{2}=0.9999, \mathrm{LOD}=0.21 \mu \mathrm{g} / \mathrm{mL} ; \mathrm{LOQ}=0.71 \mu \mathrm{g} / \mathrm{mL}\right.$, peaks 8, 9, 10, 11, 13, 14 and 18). a, b,c,d: In each line, different letters represent statistically significant differences $(p<0.05)$. $p$-value resulting from the Student $t$ test: $<0.001$ (peak 3); $<0.001$ (peak 5).

\subsection{Bioactive Properties}

\subsubsection{Antioxidant Activity}

The antioxidant activity of the hydroethanolic extracts of the seasoning mixtures was assessed through the determination of their ability to inhibit lipid peroxidation and oxidative hemolysis. For that purpose, two in vitro assays were performed: TBARS and OxHLIA, respectively. The results obtained were presented as IC 50 values $(\mu \mathrm{g} / \mathrm{mL})$, which correspond to the extract concentration needed to achieve $50 \%$ inhibition of lipid peroxidation and oxidative hemolysis, respectively. The lower the $\mathrm{IC}_{50}$ value, the greater the antioxidant and antihemolytic activity of the extracts. The results are shown in Table 8, where significant differences between the evaluated extracts are expressed. The extracts from the mixtures for meat and salad presented higher antioxidant activity, with $\mathrm{IC}_{50}$ values of $4.8 \mu \mathrm{g} / \mathrm{mL}$ and $6.6 \mu \mathrm{g} / \mathrm{mL}$, respectively. The remaining extracts also demonstrated the ability to inhibit lipid peroxidation, but higher concentrations were required to obtain the same effect, with a maximum of $27 \mu \mathrm{g} / \mathrm{mL}$ for the mixture for poultry. Also, in the OxHLIA assay, the mixture for meat extract demonstrated the ability to protect $50 \%$ of the erythrocyte population from oxidation caused by AAPH (2,2'-azobis(2-methylpropionamidine) dihydrochloride; the oxidizing agent), although for only $60 \mathrm{~min}$, with an $\mathrm{IC}_{50}$ value of $13 \mu \mathrm{g} / \mathrm{mL}$, showing no activity at $120 \mathrm{~min}$. It is also important to highlight that the mixture for poultry revealed activity at lower concentrations than Trolox $(21.8 \mu \mathrm{g} / \mathrm{mL})$, the positive 
control, with an $\mathrm{IC}_{50}$ value of $16.5 \mu \mathrm{g} / \mathrm{mL}$, similar to that of the mixture for meat. The same antioxidant capacity was verified for the other extracts, although in higher concentrations, with a delay of $60 \mathrm{~min}$ in hemolysis. However, in this case, the mixture for fish was the one that needed the highest concentration to express some activity $\left(\mathrm{IC}_{50}\right.$ of $\left.106 \mu \mathrm{g} / \mathrm{mL}\right)$. The mixture for salad extract showed an $\mathrm{IC}_{50}$ value higher than that of Trolox, but it was also the only extract revealing the capacity to delay the oxidative hemolysis for $120 \mathrm{~min}$.

Table 8. Antioxidant activities of the condiment mixtures hydroethanolic extracts (IC50, $\mu \mathrm{g} / \mathrm{mL}$; mean $\pm \mathrm{SD}$ ).

\begin{tabular}{ccccc}
\hline & $\begin{array}{c}\text { Mixture for } \\
\text { Poultry }\end{array}$ & $\begin{array}{c}\text { Mixture for } \\
\text { Meat }\end{array}$ & $\begin{array}{c}\text { Mixture for } \\
\text { Salad }\end{array}$ & $\begin{array}{c}\text { Mixture for } \\
\text { Fish }\end{array}$ \\
\hline TBARS & $27 \pm 2^{\mathrm{c}}$ & $4.8 \pm 0.3^{\mathrm{a}}$ & $6.6 \pm 0.4^{\mathrm{a}}$ & $23 \pm 2^{\mathrm{b}}$ \\
OxHLIA 60 min & $16.5 \pm 0.8^{\mathrm{a}}$ & $13 \pm 2^{\mathrm{a}}$ & $68 \pm 2^{\mathrm{b}}$ & $106 \pm 8^{\mathrm{c}}$ \\
OxHLIA 120 min & na & na & $98 \pm 6$ & na \\
\hline
\end{tabular}

na: no activity. ${ }^{a}, \mathrm{~b}, \mathrm{c}, \mathrm{d}$ : In each line, different letters represent significant differences $(p<0.05)$. Trolox $\mathrm{IC}_{50}$ values (positive control): $5.4 \pm 0.3 \mu \mathrm{g} / \mathrm{mL}$ (TBARS); $21.8 \pm 0.3 \mu \mathrm{g} / \mathrm{mL}$ (OxHLIA $60 \mathrm{~min}$ ); $43.5 \pm 0.3 \mu \mathrm{g} / \mathrm{mL}$ (OxHLIA $120 \mathrm{~min})$.

In general, all extracts revealed a great antioxidant capacity, which, according to several authors, in plants, is mainly associated with the molecules like phenolic compounds, organic acids, and tocopherols $[7,29,46]$. Other authors also correlate the antioxidant activity found in plant extracts with apigenin, in this case present as apigenin-O-malonylpentoside-hexoside in all the extracts, in high concentrations. Apigenin is an aglycone that holds several biological assets, including antioxidant, anti-inflammatory, antitumor, antigenotoxic, antiallergic, neuroprotective, cardioprotective, and antimicrobial activities [44]. Notable biological effects are also attributed to rosmarinic acid, namely antioxidant properties [47], which is mostly present in the mixtures for poultry and meat that exhibited the greatest capacity to inhibit the oxidative hemolysis, in OxHLIA assay. Significant differences were observed in the antioxidant activity between the performed assays, mainly regarding the mixture for poultry, which, in the TBARS assay, revealed the worst ability to inhibit lipid peroxidation compared to the other extracts. Though, for OxHLIA assay, this extract proved to be one of the most effective ones, with an $\mathrm{IC}_{50}$ value lower than that of Trolox. These discrepancies may be due to the concentration of tocopherols in this mixture, which, according to our results, was lower than in the other mixtures. Tocopherols are known to have an important role in preventing lipid peroxidation, herein demonstrated by the inhibition of formation of TBARS, a direct consequence of this capacity [48]. Despite the highest $\mathrm{IC}_{50}$ values found for the extract, comparing with Trolox, it is important to note that pure compounds generally reveal more activity than extracts, especially this specific water-soluble vitamin E derivative, which holds an extraordinary antioxidant activity. Thus, the $\mathrm{IC}_{50}$ values obtained with the extracts, where each of the antioxidant compounds is present in a lower final concentration, are great results.

\subsubsection{Antimicrobial Activity}

In this study, antibacterial analysis was performed by means of eight bacterial strains of clinical interest and the results are shown in Table 9. In general, all extracts showed activity against the studied bacteria, except for Klebsiella pneumoniae and Pseudomonas aeruginosa, for which a concentration higher than $20 \mathrm{mg} / \mathrm{mL}$ would be required to exert inhibitory activity, for all the extracts. The extracts revealing the most promising results were those of the mixtures for meat and for salad, which revealed MIC values of $2.5 \mathrm{mg} / \mathrm{mL}$ for Enterococcus faecalis. This bacterium, together with Escherichia coli, revealed the highest sensitivity for the mixture of fish extract (MIC value of $5 \mathrm{~b} \mathrm{mg} / \mathrm{mL}$ ). For all the assessed bacteria, the minimum bactericidal concentration $(\mathrm{MBC})$, after further evaluation of microbial growth, was found to be higher than $20 \mathrm{mg} / \mathrm{mL}$ for all the extracts. This observation could indicate that the antimicrobial compounds present in the extracts mainly 
hold inhibitory activity, with higher concentrations required for a possible bactericidal activity. It should be noted that, in this work, microorganisms were obtained from clinical isolates, which often have greater resistance to antibiotics, compared to commercial ones.

Table 9. Antimicrobial activity (minimal inhibition concentration (MIC) and minimal bactericidal concentration (MBC); $\mathrm{mg} / \mathrm{mL}$ ) of the condiment mixtures hydroethanolic extracts.

\begin{tabular}{|c|c|c|c|c|c|c|c|c|c|c|}
\hline & \multirow{2}{*}{$\begin{array}{c}\begin{array}{c}\text { Mixture } \\
\text { for } \\
\text { Poultry }\end{array} \\
\text { MIC }\end{array}$} & \multirow{2}{*}{$\begin{array}{c}\begin{array}{c}\text { Mixture } \\
\text { for Meat }\end{array} \\
\text { MIC }\end{array}$} & \multirow{2}{*}{$\begin{array}{c}\text { Mixture } \\
\text { for } \\
\text { Salad }\end{array}$} & \multirow{2}{*}{$\begin{array}{c}\begin{array}{c}\text { Mixture } \\
\text { for } \\
\text { Fish }\end{array} \\
\text { MIC }\end{array}$} & \multicolumn{2}{|c|}{ Ampicillin * } & \multicolumn{2}{|c|}{ Imipenem * } & \multicolumn{2}{|c|}{ Vancomicin * } \\
\hline & & & & & MIC & $\mathrm{MBC}$ & MIC & MBC & MIC & MBC \\
\hline E. coli & 5 & 10 & 5 & 5 & $<0.15$ & $<0.15$ & $<0.0078$ & $<0.0078$ & $\mathrm{nt}$ & $\mathrm{nt}$ \\
\hline $\begin{array}{c}\text { K. } \\
\text { pneumoniae }\end{array}$ & $>20$ & $>20$ & $>20$ & $>20$ & 10 & 20 & $<0.0078$ & $<0.0078$ & nt & nt \\
\hline M. morganii & 5 & 2.5 & 5 & 10 & 20 & $>20$ & $<0.0078$ & $<0.0078$ & nt & $\mathrm{nt}$ \\
\hline P. mirabilis & 10 & 10 & 10 & 20 & $<015$ & $<0.15$ & $<0.0078$ & $<0.0078$ & $\mathrm{nt}$ & $\mathrm{nt}$ \\
\hline P. aeruginosa & $>20$ & $>20$ & $>20$ & $>20$ & $>20$ & $>20$ & 0.5 & 1 & nt & nt \\
\hline E. faecalis & 5 & 2.5 & 2.5 & 5 & $<0.15$ & $<0.15$ & nt & nt & $<0.0078$ & $<0.0078$ \\
\hline $\begin{array}{l}\text { L. monocyto- } \\
\text { genes }\end{array}$ & 20 & 10 & 10 & 10 & $<0.15$ & $<0.15$ & $<0.0078$ & $<0.0078$ & nt & nt \\
\hline MRSA & 5 & 2.5 & 5 & 10 & $<0.15$ & $<0.15$ & nt & nt & 0.25 & 0.5 \\
\hline
\end{tabular}

Minimum inhibitory concentration (MIC); minimum bactericidal concentration (MBC); methicillin resistant Staphylococcus aureus (MRSA); nt-not tested. * positive control.

MAPs are known to be wealthy in several compounds with antimicrobial properties, with organic acids playing an important role in this field by preserving the quality and organoleptic characteristics of fruits and vegetables [49]. In general, all extracts were effective against the bacteria under study, and no relationship between the more complex constitution of the cell wall of Gram-negative bacteria and their effectiveness was evidenced. The extracts from the mixtures for meat and salad showed, in general, the best antimicrobial activity for all strains and, considering the possible antagonistic and/or synergistic effects of the different bioactive compounds present in these mixtures, malic and oxalic acids may be the main responsible for the activity of these extracts. Previous studies have identified different organic acids from Japanese apricot fruits and determined antimicrobial activities against E. coli, Bacillus subtilis, and Staphylococcus aureus [50]. Other authors reported the antimicrobial activity of oxalic acid against nine different phytopathogenic bacteria, with a bactericidal effect [51]. In addition, phenolic compounds may also contribute to the antimicrobial activity presented by the extracts [9], where quercetin and isorhamnetin, present in greater quantities in the extracts with greater antimicrobial potential, may be those that mostly influence this bioactivity. These compounds are recognized not only for their antimicrobial activity, but also by their antioxidant, anti-inflammatory, and anticancer properties [52,53]. Although many studies seek to isolate the active components of these plants, the effect of an herbal medicine is usually explained by the synergistic action between the compounds. This interaction can occur in such a way as to enhance its antimicrobial action, or it can promote the reduction and even the loss of this activity [10].

\subsubsection{Anti-Inflammatory Activity}

The $\mathrm{IC}_{50}$ values reached by assessing the ability of the hydroethanolic extracts of the mixtures to inhibit $50 \%$ of nitric oxide (NO) production in the mouse macrophage cell line (RAW 264.7) are presented in Table 10. Generally, all the extracts showed lower efficiency than the positive control (dexamethasone), demonstrating, however, anti-inflammatory potential, except for the extract of mixture for salad, which did not show activity at the maximum tested concentration $(400 \mu \mathrm{g} / \mathrm{mL})$. The mixtures for poultry and fish showed the most effective results, with a concentration of $54 \mu \mathrm{L} / \mathrm{mL}$ and $59 \mu \mathrm{g} / \mathrm{mL}$, respectively, required to promote $50 \%$ inhibition of NO production. The mixture for salad, on the other hand, did not reveal activity at the highest tested concentration $(400 \mu \mathrm{g} / \mathrm{mL})$. 
Table 10. Anti-inflammatory and antitumor activity $\left(\mathrm{IC}_{50}\right.$ values $\left.\mu \mathrm{g} / \mathrm{mL}\right)$ of the condiment mixtures hydroethanolic extracts (mean \pm SD).

\begin{tabular}{|c|c|c|c|c|}
\hline & Mixture for Poultry & Mixture for Meat & Mixture for Salad & Mixture for Fish \\
\hline \multicolumn{5}{|c|}{ Anti-inflammatory activity } \\
\hline RAW 264.7 & $54 \pm 2^{a}$ & $149 \pm 5^{b}$ & $>400$ & $59 \pm 3^{a}$ \\
\hline \multicolumn{5}{|c|}{ Antitumor activity } \\
\hline AGS & $261 \pm 5^{c}$ & $184 \pm 12^{b}$ & $98 \pm 2^{a}$ & $301 \pm 4^{\mathrm{d}}$ \\
\hline HeLa & $292 \pm 3^{b}$ & $327 \pm 22^{c}$ & $88 \pm 1^{a}$ & $294 \pm 2^{b}$ \\
\hline MCF-7 & $183.3 \pm 0.2^{\mathrm{c}}$ & $53.25 \pm 0.02^{b}$ & $10.54 \pm 0.02^{\mathrm{a}}$ & $215 \pm 5^{d}$ \\
\hline NCI-H460 & $341.7 \pm 0.2^{b}$ & $>400$ & $318 \pm 1^{\mathrm{a}}$ & $348 \pm 2^{c}$ \\
\hline CaCo-2 & $238.9 \pm 0.5^{b}$ & $238 \pm 2^{b}$ & $192 \pm 1^{\mathrm{a}}$ & $294.01 \pm 0.03^{c}$ \\
\hline
\end{tabular}

Several authors reported that apigenin derivates have the capacity to inhibit organism's inflammatory response [44,54], which indicates that apigenin-O-malonyl-pentosidehexoside, present in high concentrations in all the studied extracts, has possibly enhanced the anti-inflammatory effect of the extracts. Additionally, luteolin, which derivatives were found in the studied extracts as luteolin-7-O-glucuronide, luteolin-7-O-glucoside, and luteolin7-O-rutinoside, is widely known for being responsible for several pharmacological activities. Its anti-inflammatory activity is partially related to the regulation of inflammatory mediators and various cytokines in in vitro and in vivo models $[55,56]$. Plants with larger amounts of luteolin have long been used in Irani, Brazil, and in traditional Chinese medicines to treat diseases related to inflammation $[57,58]$. Different types of luteolinmediated regulation of inflammatory mediators have already been described, namely in RAW 264.7 macrophages [59-61]. The lower anti-inflammatory activity observed in the mixture for meat, in which a higher content of phenolic compounds was identified, may be due to antagonistic effects between the various compounds present in the extract, although further studies would be needed to prove this hypothesis. It is worthy to remember that the countless biological activities ascribed to MAPs mostly result from the action of their diverse constituents or synergisms between them, constituting a rich ethnopharmacological heritage [10].

\subsubsection{Antitumor Activity}

The extracts obtained from the different mixtures were also tested for their antitumor activity against five tumor cell lines (Table 10). The results are expressed as $\mathrm{GI}_{50}$ values, translating the extract concentration that provides $50 \%$ of cell growth inhibition. All extracts showed a $\mathrm{GI}_{50}$ value higher than that of the positive control (elipticin). However, it was observed that, except for the mixture for meat (for the NCI-H460 line), all extracts showed effective results in inhibiting the growth of the tested cell lines. The mixture for salad showed the lowest $\mathrm{GI}_{50}$ values for all the cell lines, particularly for MCF-7 $(10.54 \mu \mathrm{g} / \mathrm{mL})$. In general, MCF-7 cell line was the most sensitive to all the tested extracts, in contrast with NCI-H460 cell line that required higher extract concentrations. Overall, the mixture for salad showed to be the most effective in inhibiting tumor growth in all the tested cell lines.

Several epidemiological studies have been consistently reporting an inverse association between the intake of phenolic compounds, particularly those derived from phenolic acids and flavonols, and the risk of cancer in humans. Subsequently, reviews on cancer prevention and treatment in animal and cell line models have been reflecting the effectiveness of phenolic compounds in inhibiting cancer development. Additionally, these compounds are described as having different mechanisms of action against tumor cells, namely through targeting human cell receptors, enzymes, transcription factors, and others [18]. Quercetin 
derivatives, widely occurring in plants and present in the studied extracts have been attracting further attention due to their anticancer activity, as well as antioxidant, antiviral, antibacterial, and anti-inflammatory effects [52]. These compounds are also known to inhibit the proliferation of various cell lines related to human breast cancer due to its pro-oxidant capacity, which contributes to the prevention of tumor growth and stimulates the induction of apoptosis, interrupting the normal cell cycle [62]. The presence of this phenolic compound in the studied extracts supports the results obtained in MCF-7 cell line, which revealed a high sensitivity to all the extracts. The luteolin derivates detected in the extracts may also be responsible for their pharmacological activities. According to previous studies, these compounds exert anticancer effects, inducing apoptosis, interrupting the cell cycle, and inhibiting metastasis and angiogenesis in several cancer cell lines, such as breast, colon, pancreas, and lung cell lines, among others [63-65]. The effects of apigenin in cancer prevention may be due to its potent antioxidant and anti-inflammatory activities, investigated in studies carried out in ovarian and breast cancer and in the risk of recurrence of neoplasia in colorectal cancer $[44,54,66,67]$. The great antitumor activity presented by the mixture for salad extract is not, however, exclusively linked to the compounds derived from quercetin and luteolin, since they are present in similar concentrations in all the studied extracts. Furthermore, this mixture has the lowest total content of phenolic compounds, which can lead to conclude that its activity is also ascribed to the remaining bioactive compounds assessed (e.g., organic acids and tocopherols). The action of these compounds, either individually or in interaction with each other, generating antagonistic, synergistic, or additive effects, make it difficult to predict their effects in trials with different cell lines [10]. Also, flavonoids can be an important complement in the prevention and treatment of several types of cancer, due to their natural origin, safety, and low cost in relation to synthetic drugs. However, as most of the findings cited in the present work are based on in vitro and in vivo studies, they do not necessarily represent this effect in humans. Thus, further investigations would be necessary to complement this study, focusing the mechanisms of action of these compounds in cancer prevention/treatment.

\section{Materials and Methods}

\subsection{Samples}

The samples of seasoning mixtures were provided by Cantinho das Aromáticas (Vila Nova de Gaia, Porto), in the dry state. Their storage was carried out in a dry place and protected from light. The mixture for poultry consists of Allium schoenoprasum L. , Petroselinum crispum L., Salvia officinalis L., Satureja montana L., and Thymus vulgaris L.; the mixture for meat is composed of P.crispum, S. officinalis, Thymus mastichina L., and Rosmarinus officinalis L.; the mixture for salads consists of A. schoenoprasum, P. crispum, Origanum vulgare L., and T. mastichina, and the mixture for fish is composed of A. schoenoprasum, Artemisia dracunculus L., P. crispum, and Thymus $\times$ citriodorus $\mathrm{L}$.

\subsection{Extract Preparation}

Hydroethanolic extractions were performed by maceration of the dry material for the analysis of the phenolic composition and bioactive properties, as previously described by Barros et al. [68]. Briefly, $1.5 \mathrm{~g}$ of sample was subjected to an extraction of $1 \mathrm{~h}\left(25^{\circ} \mathrm{C}\right.$ at $150 \mathrm{rpm}$ ) twice with $40 \mathrm{~mL}$ of ethanol (Sigma-Aldrich, St. Louis, MO, USA)/water (80:20; $v / v)$ and, then, filtered through Whatman No. 4 paper. The ethanol of the combined extracts was removed using a rotary evaporator (Büchi R-210, Flawil, Switzerland) and the extract was frozen and lyophilized (FreeZone 4.5 model 7750031, Labconco, Kansas City, MO, USA) for further analysis.

\subsection{Chemical Composition}

\subsubsection{Proximate Composition and Energetic Value}

According to the Official Methods of Analysis of AOAC [69], the proximate composition was determined and expressed in $\mathrm{g} / 100 \mathrm{~g}$ dry sample. The incineration at $550 \pm 5{ }^{\circ} \mathrm{C}$ 
was used to determine the ash content. Crude protein was estimated by the macro-Kjeldahl method $(\mathrm{N} \times 6.25)$ using an automatic distillation and titration unit (model Pro-NitroA, JP Selecta, Barcelona). Soxhlet extraction was used to determine the crude fat, with petroleum ether during $7 \mathrm{~h}$. Total carbohydrates content was calculated by difference: Total carbohydrates $(\mathrm{g} / 100 \mathrm{~g})=100-($ fat $+\mathrm{g}$ ash $+\mathrm{g}$ proteins $)$. The energetic value was calculated according to the Atwater system using the formula: Energy $(\mathrm{kcal} / 100 \mathrm{~g})=4 \times$ (g proteins $+\mathrm{g}$ carbohydrates $)+9 \times(\mathrm{g}$ fat $)$.

\subsubsection{Sugars}

The extraction of free sugars from the dry samples was carried out according to Barros et al. [68] The compounds were identified by high performance liquid chromatography with a refraction index detector (HPLC-RI; Knauer, Smartline 1000 and Smartline 2300 systems, respectively) operating as previously described by the authors. Peaks identification was performed by comparisons of their relative retention time (Rt) with authentic standards. Quantification was completed using melezitose as IS, (Sigma-Aldrich, St. Louis, MO, USA). Results were processed in a Clarity Software (Data Apex, Prague, Czech Republic) and expressed in $\mathrm{g}$ per $100 \mathrm{~g} \mathrm{dw}$.

\subsubsection{Organic Acids}

The analysis of organic acids followed the protocol established by the group [70]. The evaluation was performed by ultra-fast liquid chromatography coupled to a photodiode array detector (UFLC-PDA; Shimadzu Coperation, Kyoto, Japan). The separation of the compounds was carried out in a C18 SphereClone (Phenomenex) reverse phase column $\left(5 \mu \mathrm{m}, 250 \times 4.6 \mathrm{~mm}\right.$ id) thermostated at $35^{\circ} \mathrm{C}$, using $3.6 \mathrm{mM}$ sulfuric acid solution as an eluent at a flow rate of $0.8 \mathrm{~mL} / \mathrm{min}$. The identification was carried out by comparing the chromatograms obtained for the analyzed samples with those obtained using commercial standards. The quantification of the compounds was done by relating the peak areas, recorded at $215 \mathrm{~nm}$, with the calibration curves obtained with commercial standards for each compound. The results are presented in $\mathrm{mg}$ per $100 \mathrm{~g} \mathrm{dw}$.

\subsubsection{Tocopherols}

For the determination of tocopherols, the methodology was applied according to that previously described by the authors [68]. The previously described HPLC system was used, coupled to a fluorescence detector (FP-2020; Jasco, Japan) programmed for excitation at $290 \mathrm{~nm}$ and emission at $330 \mathrm{~nm}$. The separation of the tocopherol isoforms was achieved using a normal phase column of Polyamide II ( $250 \mathrm{~mm} \times 4.6 \mathrm{mmi}$.d.) from YMC Waters (Japan), operating at $30{ }^{\circ} \mathrm{C}$. The mobile phase used was a mixture of hexane and ethyl acetate (7: 3, v/v) with a flow rate of $1 \mathrm{~mL} / \mathrm{min}$ and an injection volume of $20 \mu \mathrm{L}$. Quantification was based on the response of the fluorescence signal, using the internal standard method and by chromatographic comparison with standards. Tocol (Matreya, Pleasant Gap, State College, PA, USA) was used as internal standard, and the results were expressed in $\mathrm{mg} / 100 \mathrm{~g} \mathrm{dw}$.

\subsubsection{Fatty Acids}

The fatty acids were determined after the trans-esterification process, as previously described by Barros et al. [68]. The analysis was made using a gas chromatographer DANI model GC 1000 instrument equipped with a split/splitless injector and a flame ionization detector (GC-FID, $260{ }^{\circ} \mathrm{C}$ ). The identification and quantification of the compounds were performed by comparing the relative retention times of fatty acid methyl ester (FAME) from commercial standards. Fatty acids were processed using Clarity Software (DataApex 4.0, Prague, Czech Republic) and the results expressed in relative percentage. 


\subsubsection{Phenolics Compounds}

The phenolic compounds were evaluated in the lyophilized hydroethanolic extracts and redissolved in ethanol/water $(80: 20 ; v / v)$ to a final concentration of $10 \mathrm{mg} / \mathrm{mL}$. The evaluation was performed using a Dionex Ultimate 3000 UPLC (Thermo Scientific, San Jose, CA, USA), equipped with a DAD detector (280 and $370 \mathrm{~nm}$ as the preferred wavelength) and coupled to an electrospray ionization mass detector (LC-DAD-ESI/MSn). The chromatographic separation of the compounds was performed with a Waters Spherisorb S3 ODS-2 C18 column ( $3 \mu \mathrm{m}, 4.6 \mathrm{~mm} \times 150 \mathrm{~mm}$, Waters, Milford, MA, USA), operating at $35{ }^{\circ} \mathrm{C}$. The elution solvents, working in the gradient, were $0.1 \%$ formic acid in water and acetonitrile. Finally, for detecting MS in negative mode, a Linear Ion Trap LTQ XL mass spectrometer (ThermoFinnigan, San Jose, CA, USA) equipped with an electrospray ionization source (ESI) was used. The identification of phenolic compounds was performed based on chromatographic behavior, spectra, and UV-Vis masses, by comparison with standard compounds or data previously described in the literature, using the Xcalibur ${ }^{\circledR}$ software (ThermoFinnigan, San Jose, CA, USA). Quantitative analysis of the identified compounds was performed using calibration curves based on the UV signal of the standard compounds. When commercial standards were not available, the calibration curves of the most similar standards were used. The operating conditions were previously described in detail by Bessada et al. [71] as well as the identification and quantification procedures. The results are expressed in $\mathrm{mg} / \mathrm{g}$ extract.

\subsection{Bioactive Properties}

\subsubsection{Antioxidant Activity}

The antioxidant activity of the extracts obtained from the four mixtures was evaluated by means of two cell assays: oxidative hemolysis inhibition assays (OxHLIA) and the lipid peroxidation inhibition by thiobarbituric acid reactive substances assay (TBARS), according to the procedure reported in detail by Lockowandt et al. [72]. The results were expressed in $\mathrm{IC}_{50}$ values, meaning the concentration of extract $(\mu \mathrm{g} / \mathrm{mL})$ necessary to prevent oxidative hemolysis of $50 \%$ of the erythrocytes population for a $\Delta \mathrm{t}$ of 60 and $120 \mathrm{~min}$ and to prevent lipid peroxidation, respectively. Trolox (Sigma-Aldrich, St. Louis, MO, USA) was the positive control used.

\subsubsection{Antimicrobial Activity}

The antibacterial activity of the extracts was determined by redissolving them in water to obtain a $100 \mathrm{mg} / \mathrm{mL}$ stock solution, being subsequently subjected to successive dilutions. The microdilution method [73] was used against microorganisms from clinical isolates of patients hospitalized in various departments of the Local Health Unit of Bragança and Centro Hospitalar de Trás-os-Montes and Alto-Douro Vila Real, Northeast Portugal. Five Gram-negative bacteria (Escherichia coli, Klebsiella pneumoniae, Morganella morganii, Proteus mirabilise, and Pseudomonas aeruginosa) and three Gram-positive bacteria (Enterococcus faecalis, Listeria monocytogenese, and methicillin-resistant Staphylococcus aureus (MRSA)) were used. The results were presented as minimal inhibition concentrations (MICs) and minimal bactericidal concentrations (MBCs). Ampicillin was used as a positive control for all bacterial strains, Imipenemo for all Gram-negative bacteria tested and L. monocytogenes and Vancomycin for Enterococcus faecalis and MRSA.

\subsubsection{Anti-Inflammatory Activity}

The extracts were redissolved in water at a concentration of $8 \mathrm{mg} / \mathrm{mL}$ and then diluted in the range of 400 to $6.25 \mu \mathrm{g} / \mathrm{mL}$. A mouse macrophage-like cell line RAW 264.7 was used in this study, the inflammation was induced by LPS, and the Griess Reagent System (GRS) kit was applied to determine the nitric oxide, measured at $540 \mathrm{~nm}(\mathrm{EL} \times 800$ microplate reader, Bio-Tek Instruments, Inc; Winooski, VT, USA), as described previously [74]. The results were expressed in $\mathrm{IC}_{50}$ values (sample concentration providing $50 \%$ of inhibition 
of NO production, $\mu \mathrm{g} / \mathrm{mL})$ and Dexamethasone $(50 \mu \mathrm{M})$ was used as a positive control, while in negative controls, no LPS was added.

\subsubsection{Antitumor Activity}

The extracts were re-dissolved in water at a concentration of $8 \mathrm{mg} / \mathrm{mL}$ and then diluted in the range of 400 to $6.25 \mu \mathrm{g} / \mathrm{mL}$. The antitumoral activity was evaluated in five human tumor cell lines: AGS (gastric adenocarcinoma), CaCo-2 (colorectal adenocarcinoma) HeLa (cervical adenocarcinoma), MCF-7 (breast adenocarcinoma), and NCI-H460 (lung carcinoma). The cell lines were plated in 96-well plates, with a final density of $1.0 \times 10^{4}$ cells $/ \mathrm{mL}$ and were allowed to attach for $24 \mathrm{~h}$. Next, different extract concentrations were added to the cells, which were incubated for $48 \mathrm{~h}$. Both cells treatment and the Sulforhodamine B assay were carried out according to a protocol established by Abreu et al. [75]. All results were expressed as the sample concentration inhibiting $50 \%$ of the net cell growth (GI50 values, $\mu \mathrm{g} / \mathrm{mL}$ ). Ellipticine (Sigma-Aldrich, St. Louis, MO, USA) was applied as the positive control.

\subsection{Statistical Analysis}

The described tests were performed in triplicate, the results being expressed as mean values \pm SD. An analysis of variance (ANOVA) was performed based on the Tukey test with $\alpha=0.05$ (when the homoscedasticity of the distributions was verified) or the Tamhane T2 test (heteroscedastic distributions) to classify the statistical differences between the different parameters evaluated. Compliance with ANOVA requirements, specifically the normal distribution of results and the homogeneity of variances, was verified using the Shapiro-Wilk test and the Levene test, respectively. In the remaining cases, the t-Student test was applied, considering a value of $\alpha=0.05$ ( $95 \%$ confidence). IBM SPSS Statistics for Windows, version 22.0, was used (IBM Corp., Armonk, NY, USA).

\section{Conclusions}

The results obtained in this study suggest that the studied condiment mixtures are an excellent source of bioactive compounds, supporting the hypothesis that specific phenolic compounds, as well as organic acids and tocopherols, strongly contribute to the bioactive properties of the extracts. Their phenolic composition, especially in apigenin, luteolin, and quercetin derivates confer them high antioxidant and anti-inflammatory capacity and may also be associated with the antitumor and antimicrobial properties against a diversity of microorganisms. Given the wealth of these plant mixtures in valuable bioactive compounds with beneficial effects on human health, their inclusion in daily human diet, as well as their application in food industry, for instance for preserving purposes, is of great importance. Several beneficial effects can be achieved considering the possible cumulative and synergistic effects of all the identified bioactive compounds.

Author Contributions: Conceptualization, C.P., I.C.F.R.F. and L.B.; data curation, C.P. and L.B.; formal analysis, C.P. and Â.L.; funding acquisition, I.C.F.R.F. and L.B.; investigation, C.N., A.K.M., M.I.D., M.A.-O., M.J.A. and R.C.C.; project administration, C.P., I.C.F.R.F. and L.B.; resources, I.C.F.R.F. and L.B.; supervision, C.P., I.C.F.R.F. and L.B.; validation, C.P., M.I.D. and L.B.; visualization, I.C.F.R.F. and L.B.; writing—original draft, C.N., C.P. and Â.L.; writing—review and editing, C.P., I.C.F.R.F. and L.B. All authors have read and agreed to the published version of the manuscript.

Funding: The authors are grateful to the Foundation for Science and Technology (FCT, Portugal) for financial support through national funds FCT/MCTES to CIMO (UIDB/00690/2020); national funding by F.C.T. and P.I., through the institutional scientific employment program-contract for C.P., M.I.D., R.C.C. and L.B. contracts and A.K.M. (2020.06231.BD) and M.A.-O. (2020.06297.BD) PhD grants. To FEDER-Interreg España-Portugal programme for financial support through the project TRANSCoLAB 0612_TRANS_CO_LAB_2_P; to ERDF through the Regional Operational Program North 2020, within the scope of Project GreenHealth-Norte-01-0145-FEDER-000042.

Institutional Review Board Statement: Not applicable. 
Informed Consent Statement: Informed consent was obtained from all subjects involved in the study.

Data Availability Statement: The data presented in this study are available in this article.

Acknowledgments: The authors are grateful to the company "Cantinho das Aromáticas, Viveiros, Lda" for samples supply.

Conflicts of Interest: The authors declare no conflict of interest.

Sample Availability: Samples of the condiment mixtures are available from the authors.

\section{References}

1. INFARMED. Infarmed 15 Anos: Olhar o Passado, Projectar o Futuro; INFARMED—Autoridade Nacional do Medicamento e Produtos de Saúde, I.P. Ministério da Saúde: Lisboa, Portugal, 2008; pp. 101-102. ISBN 978-972-8425-90-6.

2. Tascilar, M.; Floris, A.J.; Verweij, J.; Mathijssen, R.H.J. Complementary and alternative medicine during cancer treatment: Beyond innocence. Oncologist 2006, 7, 732-741. [CrossRef]

3. Cunha, M.; Martins, R.; André, S.; Albuquerque, C.; Cunha, B.; Almeida, D.; Silva, M.; Gaspar, R.; Fonseca, S. Estudantes $28^{\circ}$ CLE ESSV IPV. Consumo de sal, açúcar, ervas/plantas aromáticas e especiarias. Servir 2016, 59, 36-41.

4. Pereira, C.; Barros, L.; Ferreira, I.C.F.R. A comparison of the nutritional contribution of thirty-nine aromatic plants used as condiments and/or herbal infusions. Plant Foods Hum. Nutr. 2015, 70, 176-183. [CrossRef]

5. Pereira, C.; Barros, L.; Ferreira, I.C.F.R. Plantas aromáticas usadas como condimentos: Prevalência de ácidos gordos polinsaturados. Rev. Ciências Agrárias 2017, 40, 155-159. [CrossRef]

6. Shahidi, F.; Peng, H. Bioaccessibility and bioavailability of phenolic compounds. J. Food Bioact. 2018, 4, 11-68. [CrossRef]

7. Ferreira, I.C.F.R.; Martins, N.; Barros, L. Phenolic Compounds and Its Bioavailability: In Vitro Bioactive Compounds or Health Promoters? Adv. Food Nutr. Res. 2017, 82, 1-44.

8. Sytar, O.; Hemmerich, I.; Zivcak, M.; Rauh, C.; Brestic, M. Comparative analysis of bioactive phenolic compounds composition from 26 medicinal plants. Saudi J. Biol. Sci. 2018, 25, 631-641. [CrossRef]

9. Tungmunnithum, D.; Thongboonyou, A.; Pholboon, A.; Yangsabai, A. Flavonoids and Other Phenolic Compounds from Medicinal Plants for Pharmaceutical and Medical Aspects: An Overview. Medicines 2018, 5, 93. [CrossRef] [PubMed]

10. Cunha, A.; Nogueira, M.; Roque, O. Plantas Aromáticas e óleos Essenciais: Composição e Aplicações; Fundação Calouste Gulbenkian: Lisboa, Portgal, 2012.

11. Farrell, K. Spices, Condiments and Seasonings, 2nd ed.; Springer: Berlin/Heidelberg, Germany, 1999; pp. 3-5.

12. Pereira, C.; Barreira, J.; Calhelha, R.; Queiroz, M.; Barros, L.; Ferreira, I.C.F.R. New insights into the effects of formulation type and compositional mixtures on the antioxidant and cytotoxic activities of dietary supplements based-on hepatoprotective plants. Food Funct. 2014, 5, 2052-2060. [CrossRef]

13. Ribeiro, A.; Barros, L.; Calhelha, R.C.; Carocho, M.; Ćirić, A.; Soković, M.; Dias, M.; Santos-Buelga, C.; Barreiro, M.F.; Ferreira, I.C.F.R. Tarragon extract as a functional ingredient for development of new pizza dough: Comparative performance with ascorbic acid (E300). J. Funct. Foods 2016, 26, 268-278. [CrossRef]

14. Dias, M.I.; Barros, L.; Carvalho, A.M.; Alves, R.C.; Oliveira, M.B.P.P.; Ferreira, I.C.F.R. Caracterização química de amostras silvestres e comerciais de Achillea millefolium L. Rev. Ciências Agrárias 2017, 40, 151-160.

15. Takwa, S.; Caleja, C.; Barreira, J.; Soković, M.; Achour, L.; Barros, L.; Ferreira, I.C.F.R. Arbutus unedo L. and Ocimum basilicum L. as sources of natural preservatives for food industry: A case study using loaf bread. LWT-Food Sci. Technol. 2018, 88, 47-55. [CrossRef]

16. Mishra, S.; Rao, C.; Ojha, S.; Vijayakumar, M.; Verma, A.; Alok, S. An analytical review of plants for antidiabetic activity with their phytoconstituent \& mechanism of action. Int. J. Pharm. Sci. Res. 2010, 1, 29-46.

17. Vaz, J.; Almaida, G.; Ferreira, I.; Martins, A.; Vasconcelos, M. Clitocybe alexandri extract induces cell cycle arrest and apoptosis in a lung cancer cell line: Identification of phenolic acids with cytotoxic potential. Food Chem. 2012, 132, 482-486. [CrossRef]

18. Falcão, S.I.; Calhelha, R.C.; Touzani, S.; Lyoussi, B.; Ferreira, I.C.F.R.; Vilas-Boas, M. In vitro interactions of moroccan propolis phytochemical's on human tumor cell lines and anti-inflammatory properties. Biomolecules 2019, 9, 315. [CrossRef] [PubMed]

19. Guimarães, R.; Barros, L.; Carvalho, A.; Ferreira, I.C.F.R. Infusions and decoctions of mixed herbs used in folk medicine: Synergism in antioxidant. Phytother. Res 2011, 25, 1209-1214. [CrossRef] [PubMed]

20. Taofiq, O.; Calhelha, R.C.; Heleno, S.B.; Martins, A.; Santos-Buelga, C.; Queiroz, M.; Ferreira, I. The contribution of phenolic acids to the anti-inflammatory activity of mushrooms: Screening in phenolic extracts, individual parent molecules and synthesized glucuronated and methylated derivatives. Food Res. Int. 2015, 76, 821-827. [CrossRef] [PubMed]

21. Caleja, C.; Finimundy, T.; Pereira, C.; Barros, L.; Calhelha, R.; Sokovic, M.; Ivanov, M.; Carvalho, A.M.; Rosa, E.; Ferreira, I.C.F.R. Challenges of traditional herbal teas: Plant infusions and their mixtures with bioactive properties. Food Funct. 2019, 10, 5939-5951. [CrossRef]

22. Probst, I.; Sforcin, J.; Rall, V.; Fernandes, A.; Fernandes, J. Antimicrobial activity of propolis and essential oils and synergism between these natural products. J. Venom. Anim. Toxins Incl. Trop. Dis. 2011, 17, 159-167. [CrossRef] 
23. Shang, N.; Chaplot, S.; Wu, J. 12-Food proteins for health and nutrition. In Proteins in Food Processing, 2nd ed.; Rickey, Y., Ed.; Woodhead Publishing: Cambridge, UK, 2018; pp. 301-336.

24. Saleh, A.; Selim, S.; Jaouni, S.; AbdElgawad, H. $\mathrm{CO}_{2}$ enrichment can enhance the nutritional and health benefits of parsley (Petroselinum crispum L.) and dill (Anethum graveolens L.). Food Chem. 2018, 269, 519-526. [CrossRef]

25. Pereira, C.; Barros, L.; Carvalho, A.; Ferreira, I.C.F.R. Use of UFLC-PDA for the Analysis of Organic Acids in Thirty-Five Species of Food and Medicinal Plants. Food Anal. Methods 2013, 6, 1337-1344. [CrossRef]

26. Tang, X.; Liu, J.; Dong, W.; Li, P.; Li, L.; Lin, C.; Li, D. The cardioprotective effects of citric acid and L-malic acid on myocardial ischemia/reperfusion injury. Evid. Based Complementary Altern. Med. 2013, 2013, 820695. [CrossRef]

27. Varì, R.; D'Archivio, M.; Filesi, C.; Carotenuto, S.; Scazzocchio, B.; Santangelo, C.; Masella, R. Protocatechuic acid induces antioxidant/detoxifying enzyme expression through JNK-mediated Nrf2 activation in murine macrophages. J. Nutr. Biochem. 2011, 22, 409-417. [CrossRef] [PubMed]

28. Delgado, T.; Ramalhosa, E.; Pereira, J.A.; Casal, S. Organic acid profile of chestnut (Castanea sativa Mill.) as affected by hot air convective drying. Int. J. Food Prop. 2018, 21, 557-565. [CrossRef]

29. Bouziane, A.; Bakchiche, B.; Dias, M.I.; Barros, L.; Ferreira, I.C.F.R.; AlSalamat, H.A.; Bardaweel, S.K. Phenolic compounds and bioactivity of Cytisus villosus Pourr. Molecules 2018, 23, 1994. [CrossRef] [PubMed]

30. Barros, L.; Heleno, S.; Carvalho, A.; Ferreira, I. Lamiaceae often used in Portuguese folk medicine as a source of powerful antioxidants: Vitamins and phenolics. LWT-Food Sci. Technol. 2010, 43, 544-550. [CrossRef]

31. Ribeiro-Santos, R.; Carvalho-Costa, D.; Cavaleiro, C.; Costa, H.; Albuquerque, T.; Castilho, M.; Ramos, F.; Melo, N.; Sanches-Silva, A. A novel insight on an ancient aromatic plant: The rosemary (Rosmarinus officinalis L.). Trends Food Sci. Technol. 2015, 45, 355-368. [CrossRef]

32. Zingg, J.M.; Negis, Y.; Meydani, M.; Azzi, A. Molecular mechanism of alpha-tocopherol action. In Proceedings of the 14th biennial meeting of the Society for Free Radical Research International, Beijing, China, 18 October 2008.

33. Zingg, J.M.; Libinaki, R.; Lai, C.Q.; Meydani, M.; Gianello, R.; Ogru, E.; Azzi, A. Modulation of gene expression by $\alpha$-tocopherol and $\alpha$-tocopheryl phosphate in THP-1 monocytes. Free Radic. Biol. Med. 2010, 49, 1989-2000. [CrossRef] [PubMed]

34. Petropoulos, S.A.; Fernandes, Â.; Dias, M.I.; Pereira, C.; Calhelha, R.C.; Ivanov, M.; Sokovic, M.D.; Ferreira, I.C.F.R.; Barros, L. The Effect of Nitrogen Fertigation and Harvesting Time on Plant Growth and Chemical Composition of Centaurea raphanina subsp. mixta (DC.) Runemark. Molecules 2020, 25, 3175. [CrossRef]

35. Clifford, M.N. Miscellaneous phenols in foods and beverages-Nature, occurrence and dietary burden. J. Sci. Food Agric. 2000, 80, 1126-1137. [CrossRef]

36. Clifford, M.N.; Zheng, W.; Kuhnert, N. Profiling the chlorogenic acids of aster by HPLC-MSn. Phytochem. Anal. 2006, 17, 384-393. [CrossRef]

37. Pires, T.C.S.P.; Dias, M.I.; Barros, L.; Calhelha, R.C.; Alves, M.J.; Oliveira, M.B.P.P.; Santos-Buelga, C.; Ferreira, I.C.F.R. Edible flowers as sources of phenolic compounds with bioactive potential. Food Res. Int. 2018, 105, 580-588. [CrossRef] [PubMed]

38. Jaiswal, R.; Halabi, E.A.; Karar, M.G.E.; Kuhnert, N. Identification and characterization of the phenolics of Ilex glabra L. Gray (Aquifoliaceae) leaves by liquid chromatography tandem mass spectrometry. Phytochemistry 2014, 106, 141-155. [CrossRef]

39. Barros, L.; Dueñas, M.; Dias, M.I.; Sousa, M.J.; Santos-Buelga, C.; Ferreira, I.C.F.R. Phenolic profiles of cultivated, in vitro cultured and commercial samples of Melissa officinalis L. infusions. Food Chem. 2013, 136, 1-8. [CrossRef] [PubMed]

40. Kaiser, A.; Carle, R.; Kammerer, D.R. Effects of blanching on polyphenol stability of innovative paste-like parsley (Petroselinum crispum (Mill.) Nym ex A. W. Hill) and marjoram (Origanum majorana L.) products. Food Chem. 2013, 138, $1648-1656$.

41. Tahir, N.I.; Shaari, K.; Abas, F.; Parveez, G.K.A.; Ishak, Z.; Ramli, U.S. Characterization of apigenin and luteolin derivatives from oil palm (Elaeis guineensis Jacq.) Leaf using LC-ESI-MS/MS. J. Agric. Food Chem. 2012, 60, 11201-11210. [CrossRef]

42. Lachowicz, S.; Kolniak-Ostek, J.; Oszmiański, J.; Wiśniewski, R. Influence of Maturity on the Content of Phenolic Compounds of Alium ursinum L. J. Food Process. Preserv. 2017, 41, e13089. [CrossRef]

43. Ali, F.; Naz, F.R.; Jyoti, S.; Siddique, Y. Health functionality of apigenin: A review. Int. J. Food Prop. 2017, 20, 1197-1238. [CrossRef]

44. Epifanio, N.; Cavalcanti, L.; dos Santos, K.; Duarte, P.; Kachlicki, P.; Ożarowski, M.; Riger, C.; Chaves, D. Chemical characterization and in vivo antioxidant activity of parsley (Petroselinum crispum) aqueous extract. Food Funct. 2020, 11, 5346-5356. [CrossRef]

45. Ferreira, I.C.F.R.; Barros, L.; Abreu, R.M. Antioxidants in Wild Mushrooms. Curr. Med. Chem. 2009, 16, 1543-1560. [CrossRef]

46. Morales, P.; Ferreira, I.; Carvalho, A.; Sanchez-Mata, M.; Camara, M.; Fernandez-Ruiz, V.; de Santayana, M.; Tardio, J. Mediterranean non-cultivated vegetables as dietary sources of compounds with antioxidant. LWT-Food Sci. Technol. 2014, 55, 389-396. [CrossRef]

47. Nadeem, M.; Imran, M.; Gondal, T.; Imran, A.; Shahbaz, M.; Amir, R.; Sajid, M.; Qaisrani, T.; Atif, M.; Hussain, G.; et al. Therapeutic Potential of Rosmarinic Acid: A Comprehensive Review. Appl. Sci. 2019, 9, 3139. [CrossRef]

48. Liberal, Â.; Pereira, C.; Adega, F.; Barros, L.; Ferreira, I. Tocopherols as bioactive compounds in different cultivars of Gomphrena globosa L. In Proceedings of the 2nd Symposium on Medicinal Chemistry of University of Minho, Braga, Portugal, 8 May 2015.

49. Robles, A.; Fabjanowicz, M.; Chmiel, T.; Płotka-Wasylka, J. Determination and identification of organic acids in wine samples. Problems and challenges. TrAC Trends Anal. Chem. 2019, 120, 115630. [CrossRef]

50. Gao, Z.; Shao, J.; Sun, H.; Zhong, W.; Zhuang, W.; Zhang, Z. Evaluation of different kinds of organic acids and their antibacterial activity in Japanese Apricot fruits. Afr. J. Agric. Res. 2012, 7, 4911-4918. [CrossRef] 
51. Fernandes, Â.; Petrović, J.; Stojković, D.; Barros, L.; Glamočlija, J.; Soković, M.; Martins, A.; Ferreira, I.C.F.R. Polyporus squamosus (Huds.) Fr from different origins: Chemical characterization, screening of the bioactive properties and specific antimicrobial effects against Pseudomonas aeruginosa. LWT Food Sci. Technol. 2016, 69, 91-97. [CrossRef]

52. Wang, W.; Sun, C.; Mao, L.; Ma, P.; Liu, F.; Yang, J.; Gao, Y. The biological activities, chemical stability, metabolism and delivery systems of quercetin: A review. Trends Food Sci. Technol. 2016, 56, 21-38. [CrossRef]

53. Gong, G.; Guan, Y.-Y.; Zhang, Z.-L.; Rahman, K.; Wang, S.-J.; Zhou, S.; Luan, X.; Zhang, H. Isorhamnetin: A review of pharmacological effects. Biomed. Pharmacother. 2020, 128, 110301. [CrossRef] [PubMed]

54. Salehi, B.; Venditti, A.; Sharifi-Rad, M.; Kregiel, D.; Sharifi-Rad, J.; Durazzo, A.; Lucarini, M.; Santini, A.; Souto, E.; Novellino, E.; et al. The Therapeutic Potential of Apigenin. Int. J. Mol. Sci. 2019, 20, 1305. [CrossRef] [PubMed]

55. Seelinger, G.; Merfort, I.; Schempp, C. Anti-oxidant, anti-inflammatory and anti-allergic activities of luteolin. Planta Med. 2008, 74, 1667-1677. [CrossRef] [PubMed]

56. Turner, M.; Nedjai, B.; Hurst, T.; Pennington, D. Cytokines and chemokines: At the crossroads of cell signalling and inflammatory disease. Biochim. Biophys. Acta Mol. Cell Res. 2014, 1843, 2563-2582. [CrossRef]

57. Farzaei, M.; Abbasabadi, Z.; Ardekani, M.; Rahimi, R.; Farzaei, F. Parsley: A review of ethnopharmacology, phytochemistry and biological activities. J. Tradit. Chin. Med. 2013, 33, 815-826. [CrossRef]

58. Ferrari, F.; Ferreira, L.; Souza, M.; Grabe-Guimarães, A.; Paula, C.; Rezende, S.; Saúde-Guimarães, D. Anti-Inflammatory sesquiterpene lactones from Lychnophora trichocarpha Spreng. (Brazilian arnica). Phytother. Res. 2013, 27, 384-389. [CrossRef]

59. Choi, J.; Islam, M.; Ali, M.; Kim, Y.; Park, H.; Sohn, H.; Jung, H. The effects of C-glycosylation of luteolin on its antioxidant, anti-Alzheimer's disease, anti-diabetic, and anti-inflammatory activities. Arch. Pharm. Res. 2014, 37, 1354-1363. [CrossRef] [PubMed]

60. Liu, C.; Lin, H.; Yang, D.; Chen, S.; Tseng, J.; Chang, T.; Chang, Y. Luteolin inhibits viral-induced inflammatory response in RAW264.7 cells via suppression of STAT1/3 dependent NF-kappaB and activation of HO-1. Free Radic. Biol. Med. 2016, 95, 180-189. [CrossRef]

61. Funaro, A.; Wu, X.; Song, M.; Zheng, J.G.; Rakariyatham, K.; Rodriguez-Estrada, M.; Xiao, X. Enhanced anti-inflammatory activities by the combination of luteolin and tangeretin. J. Food Sci. 2016, 81, H1320-H1327. [CrossRef]

62. Ezzati, M.; Yousefi, B.; Velaei, K.; Safa, A. A review on anti-cancer properties of Quercetin in breast cancer. Life Sci. 2020, 248, 117463. [CrossRef]

63. Imran, M.; Rauf, A.; Abu-Izneid, T.; Nadeem, M.; Shariati, M.; Khan, I.; Imran, A.; Orhan, I.; Rizwan, M.; Atif, M.; et al. Luteolin, a flavonoid, as an anticancer agent: A review. Biomed. Pharmacother. 2019, 112, 108612. [CrossRef] [PubMed]

64. Cook, M.; Liang, Y.; Besch-Williford, S.; Goyette, C.; Mafuvadze, B.; Hyder, S. Luteolin inhibits progestin-dependent angiogenesis, stem cell-like characteristics, and growth of human breast cancer xenografts. Springerplus 2015, 4, 444. [CrossRef]

65. Park, S.; Ham, S.; Kwon, T.; Kim, M.; Lee, D.; Kang, J.; Oh, S.R.; Yoon, D. Luteolin induces cell cycle arrest and apoptosis through extrinsic and intrinsic signaling pathways in MCF-7 breast cancer cells. J. Environ. Pathol. Toxicol. Oncol. 2014, 33, $219-231$. [CrossRef] [PubMed]

66. Guimarães, R.; Calhelha, R.; Froufe, H.; Abreu, R.; Carvalho, A.; Queiroz, M.; Ferreira, I.C.F.R. Wild Roman chamomile extracts and phenolic compounds: Enzymatic assays and molecular modelling studies with VEGFR-2 tyrosine kinase. Food Funct. 2016, 7, 79-83. [CrossRef] [PubMed]

67. Ribeiro, A.; Oliveira, F.; Calhelha, R.; Junior, B.; Barreiro, M.; Ferreira, I.C.F.R. Antiangiogenic potential of yogurts added with extracts rich in apigenin derivatives. In Proceedings of the $5^{\circ}$ Encontro Português de Jovens Químicos (PYCheM) e o $1^{\circ}$ Encontro Europeu de Jovens Químicos (EYCHeM), Guimarães, Portugal, 26 April 2016.

68. Barros, L.; Pereira, E.; Calhelha, R.C.; Dueñas, M.; Carvalho, A.; Santos-Buelga, C.; Ferreira, I. Bioactivity and chemical characterization in hydrophilic and lipophilic compounds of Chenopodium ambrosioides L. J. Funct. Foods 2013, 4, 1732-1740. [CrossRef]

69. AOAC Official Methods of Analysis of AOAC International. Official Methods of Analysis of AOAC International; Horwitz, W., Latimer, G., Eds.; AOAC International: Gaithersburg, MD, USA, 2016.

70. Barros, L.; Pereira, C.; Ferreira, I.C.F.R. Optimized analysis of organic acids in edible mushrooms from Portugal by ultra-fast liquid chromatography and photodiode array detection. Food Anal. Methods 2013, 6, 309-316. [CrossRef]

71. Bessada, S.M.; Barreira, J.C.; Barros, L.; Ferreira, I.C.F.R.; Oliveira, M. Phenolic profile and antioxidant activity of Coleostephus myconis (L.) Rchb.f.: An underexploited and highly disseminated species. Ind. Crops Prod. 2016, 89, 45-51. [CrossRef]

72. Lockowandt, L.; Pinela, J.; Roriz, C.; Pereira, C.; Abreu, R.; Calhelha, R.; Alves, M.; Barros, L.; Bredol, M.; Ferreira, I.C.F.R. Chemical features and bioactivities of cornflower (Centaurea cyanus L.) capitula: The blue flowers and the unexplored non-edible part. Ind. Crops Prod. 2019, 128, 496-503. [CrossRef]

73. Pires, T.; Dias, M.; Barros, L.; Alves, M.; Oliveira, M.; Santos-Buelga, C.; Ferreira, I.C.F.R. Antioxidant and antimicrobial properties of dried Portuguese apple variety (Malus domestica Borkh. Cv Bravo de Esmolfe). Food Chem. 2018, 240, 701-706. [CrossRef] [PubMed]

74. Corrêa, R.; de Souza, A.; Calhelha, R.; Barros, L.; Glamoclija, J.; Sokovic, M.; Peralta, R.; Bracht, A.; Ferreira, I.C.F.R. Bioactive formulations prepared from fruiting bodies and submerged culture mycelia of the Brazilian edible mushroom Pleurotus ostreatoroseus Singer. Food Funct. 2015, 6, 2155-2164. [CrossRef] [PubMed]

75. Abreu, R.M.; Ferreira, I.C.F.R.; Calhelha, R.C.; Lima, R.T.; Vasconcelos, M.H.; Adega, F.; Chaves, R.; Queiroz, M.J. Antihepatocellular carcinoma activity using human HepG2 cells and hepatotoxicity of 6-substituted methyl 3-aminothieno[3,2b]pyridine-2-carboxylate Derivatives: In Vitro Evaluation, Cell Cycle Analysis and QSAR Studies. Eur. J. Med. Chem. 2011, 46, 5800-5806. [CrossRef] 